\begin{tabular}{|c|l|}
\hline Title & Global existence of nonlinear elastic waves \\
\hline Author(s) & Agemi, R. \\
\hline Citation & Hokkaido University Preprint Series in Mathematics, 463, 1-57 \\
\hline Issue Date & 1999-7-1 \\
\hline DOI & 10.14943/83609 \\
\hline Doc URL & http://hdl.handle.net/2115/69213 \\
\hline Type & bulletin (article) \\
\hline File Information & pre463.pdf \\
\hline
\end{tabular}

Instructions for use 


\section{Global existence of Nonlinear Elastic Waves}

Rentaro AGEMI

Series \#463. July 1999 


\section{Introduction}

The motion for the displacement $u=u(t, x)$ of an isotropic, homogeneous, hyperelastic material is governed by a quasilinear hyperbolic system,

$$
\partial_{t}^{2} u-c_{2}^{2} \Delta u-\left(c_{1}^{2}-c_{2}^{2}\right) \operatorname{grad} \operatorname{div} u=F\left(\nabla u, \nabla^{2} u\right),
$$

in three space dimensions. The material constants $c_{1}$ and $c_{2}$ satisfy

$$
0<c_{2}<c_{1}
$$

The nonlinear term $F\left(\nabla u, \nabla^{2} u\right)$ is linear in $\nabla^{2} u$ and will be described explicitly in later sections.

It is known from the work of John [10] that the equations have almost global solutions for small initial data. Moreover, John [9] has proved that, in the spherically symmetric case, a genuine nonlinearity condition leads to the formation of singularities even for small initial data, also see [7]. Recently, Sideris [15] has proved that, for certain classes of materials satisfying a null condition, there exist global smooth solutions with small initial data. His null condition does not coincide the complement of genuine nonlinearity condition given by John [9] in the non-spherically symmetric case.

Klainerman [13] has introduced the null condition for quasilinear wave equations and has proved global existence of solutions by making use of the Lorenz invariance. John and Shatah have given in [11] an observation on the null condition, that is, the requirement that no plane wave solution of a quasilinear wave equation is genuinely nonlinear will lead to the null condition. For the systems of quasilinear wave equations with different propagation speeds, we have derived from John-Shatah observation a null condition of new type, see [1]. Moreover, Hoshiga-Kubo [4] and Yokoyama [16] have proved global existence of solutions to such systems without the benefit of Lorenz invariance.

The first aim of the present article is to derive from John-Shatah observation the null condition reflected special features of the system (1.1) which is precisely the complement of genuine nonlinearity condition given by John [9]. The second aim is to prove, 
after the characterization of the nonlinear term $F\left(\nabla u, \nabla^{2} u\right)$ by the null condition, global existence of nonlinear elastic waves without making use of the Lorenz invariance and also the scaling operator which is used in [15]. The proof is based on energy and weighted $L^{\infty}-L^{2}$ estimates. In order to establish these estiamtes, we need a new expression of fundamental solution for the linear operator in (1.1) and new commutation relations between the operators div, rot and the angular derivatives with torsion in [10].

The main theorem on global existence is stated in Section 4, after the characterization of nonlinear term by the null condition in Section 3. The null condition is derived from John-Shatah observation for general nonlinear terms containing time derivative in Section 9. The new commutation relations are given in Section 5. The weighted $L^{\infty}-L^{\infty}$ estimates for the linear operator in (1.1) is stated in Section 6 and is proved in Section 10. The main theorem will be proved in Section 8, after establishing energy and $L^{\infty}-L^{2}$ estimates in Section 7 .

\section{The equation of motion for displacement}

Let $\varphi(t, x)$ be a smooth deformation of the material evolving with time. The unknown of the problem is a displacement from the reference configuration,

$$
u(t, x)=\varphi(t, x)-x
$$

The displacement gradient is then the matrix $G=\nabla u$ with components $G_{i \ell}=\partial_{\ell} u^{i}$, where the spatial gradient will be denoted by $\nabla$ or grad. For the materials under consideration, the potential energy density is characterized by a stored energy function $\sigma=\sigma\left(\kappa_{1}, \kappa_{2}, \kappa_{3}\right)$, where $\kappa_{1}, \kappa_{2}, \kappa_{3}$ are principal invariants of the strain matrix

$$
C=G+{ }^{t} G+G^{t} G
$$

where ${ }^{t} G$ denotes the traspose of $G$. Thus the motion for the displacement is governed by a nonlinear system,

$$
\partial_{t}^{2} u-\operatorname{div} \frac{\partial \sigma}{\partial G}=0
$$


that is,

$$
\partial_{t}^{2} u^{i}-\sum_{\ell=1}^{3} \frac{\partial}{\partial x_{\ell}} \frac{\partial \sigma}{\partial G_{i \ell}}=0 \quad(i=1,2,3)
$$

for instance see $[2],[3]$.

We make use of the following formula for principal invariants:

$$
\begin{aligned}
& \kappa_{1}=\operatorname{tr} C \\
& \kappa_{2}=\frac{1}{2}\left\{(\operatorname{tr} C)^{2}-\operatorname{tr} C^{2}\right\} \\
& \kappa_{3}=\frac{1}{6}\left\{(\operatorname{tr} C)^{3}-3(\operatorname{tr} C)\left(\operatorname{tr} C^{2}\right)+2 \operatorname{tr} C^{3}\right\} .
\end{aligned}
$$

Since we will consider only small displacement, it is enough to truncate (2.2) at third order in $u$. Then relevant terms in the Taylor expansion of $\sigma$ at $\kappa_{i j}=0$ are

$$
\sigma=\sigma_{0}+\sigma_{1} \kappa_{1}+\sigma_{2} \kappa_{2}+\sigma_{3} \kappa_{3}+\frac{1}{2} \sigma_{11} \kappa_{1}^{2}+\sigma_{12} \kappa_{1} \kappa_{2}+\frac{1}{6} \sigma_{111} \kappa_{1}^{3}+\cdots
$$

Making use of the relation

$$
\frac{\partial \sigma}{\partial G}=\sum_{k=1}^{3} \frac{\partial \sigma}{\partial \kappa_{k}} \frac{\partial \kappa_{k}}{\partial G}
$$

we find from (2.1), (2.3) and (2.4) that

$$
\begin{aligned}
\frac{\partial \sigma}{\partial G}= & 2 \sigma_{1}(I+G)+4\left(\sigma_{11}+\sigma_{2}\right)(\operatorname{tr} G) I-2 \sigma_{2}\left(G+{ }^{t} G\right) \\
& +4\left(\sigma_{111}+3 \sigma_{12}+\sigma_{3}\right)(\operatorname{tr} G)^{2} I \\
& +2\left(\sigma_{11}-\sigma_{12}+\sigma_{2}-\sigma_{3}\right)\left\{2(\operatorname{tr} G) G+\operatorname{tr}\left(G^{t} G\right) I\right\} \\
& -2\left(\sigma_{12}+\sigma_{3}\right)\left\{2(\operatorname{tr} G)^{t} G+\left(\operatorname{tr} G^{2}\right) I\right\} \\
& -2\left(\sigma_{2}-\sigma_{3}\right)\left(G^{2}+G^{t} G+{ }^{t} G G\right)+2 \sigma_{3}{ }^{t} G^{t} G \\
& + \text { terms of third order and higher. }
\end{aligned}
$$

For details see [15].

We impose the condition $\sigma_{1}=0$, which implies the reference configuration is a stress-free state. The Lamé constants $\lambda, \mu$ are defined by

$$
\lambda=4\left(\sigma_{11}+\sigma_{2}\right), \mu=-2 \sigma_{2}
$$


and $\mu, \lambda+\mu$ are assumed to be positive. Set

$$
c_{1}=(\lambda+2 \mu)^{1 / 2}, c_{2}=\mu^{1 / 2} .
$$

Then it follows from (2.2), (2.5), (2.6) that the linear part of (2.2) becomes the linear hyperbolic operator

$$
L u=\partial_{t}^{2} u-c_{2}^{2} \Delta u-\left(c_{1}^{2}-c_{2}^{2}\right) \operatorname{grad} \operatorname{div} u
$$

The material constants $c_{1}$ and $c_{2}\left(c_{1}>c_{2}\right)$ correspond to the propagation speeds of longitudinal and transverse waves, respectively. Thus the truncated version of (2.2) is formulated by

$$
L u=\operatorname{div} H=F\left(\nabla u, \nabla^{2} u\right),
$$

where $H$ stands for the quadratic term in (2.5) and the last equality is the definition of $F$.

We will show that the nonlinear term $F$ has the energy symmetry. To this end, we rewrite $i$-th component of the nonlinear term $F$,

$$
F^{i}\left(\nabla u, \nabla^{2} u\right)=\sum_{j, \ell, m=1}^{3} C_{i j}^{\ell m}(\nabla u) \partial_{\ell} \partial_{m} u^{j}
$$

so that

$$
C_{i j}^{\ell m}(\nabla u)=C_{i j}^{m \ell}(\nabla u)
$$

Here

$$
C_{i j}^{\ell m}(\nabla u)=\sum_{k, n=1}^{3} C_{i j k}^{\ell m n} \partial_{n} u^{k}
$$

Then Sideris [15] has proved the energy symmetry condition.

Proposition 2.1.

$$
C_{i j}^{\ell m}(\nabla u)=C_{j i}^{\ell m}(\nabla u)
$$




\section{The characterization of nonlinear term by the null condition}

We first introduce new unknowns

$$
v(t, x)=\left(\partial_{t} u(t, x), \nabla u(t, x)\right)
$$

Then we find from (2.8) that the vector $v \in \mathbb{R}^{12}$ satisfies a quasilinear system of first order

$$
a_{0}(v) \partial_{t} v+\sum_{i=1}^{3} a_{i}(v) \partial_{i} v=0
$$

which is strongly hyperbolic near $v=0$. The precise forms of square matrices $a_{0}(v), a_{i}(v)$ of order 12 are given in Section 9. We next consider the plave wave solution $w$ of (3.1) in the form,

$$
v(t, x)=w(t, s) \quad s=\zeta \cdot x
$$

where $\zeta \cdot x$ stands for the inner product of $\zeta, x \in \mathbb{R}^{3}$. Then $w$ satisfies the following system in one space dimension

$$
a_{0}(w) \partial_{t} w+\sum_{i=1}^{3} \zeta_{i} a_{i}(w) \partial_{s} w=0
$$

In Section 9, we will define the genuien nonlinearity for (3.3) and derive the null condition from the John-Shatah observation for general nonlinear term involving time derivative. Since the nonlinear term $F$ in (2.8) does not contain time derivative, Proposition 9.1 is equivalent to

Proposition 3.1 The quasilinear system (3.3) is not genuinely nonlinear for any $\zeta \neq 0$ if and only if

$$
\sum_{i j k \ell m n=1}^{3} C_{i j k}^{\ell m n} X_{i} X_{j} X_{k} X_{\ell} X_{m} X_{n}=0 \quad \text { for } X \in \mathbb{R}^{3}
$$


and

$(\mathrm{N})_{2}$

$$
\begin{aligned}
& \sum_{i k \ell m n=1}^{3} C_{i i k}^{\ell m n}\left(|X|^{2}-X_{i}^{2}\right) \xi_{k} X_{\ell} X_{m} X_{n} \\
& -\sum_{i \neq j, k \ell m n=1}^{3} C_{i j k}^{\ell m n} X_{i} X_{j} \xi_{k} X_{\ell} X_{m} X_{n}=0 \\
& \text { for } \xi, X \in \mathbb{R}^{3} \text { satisfying } \xi \cdot X=0,
\end{aligned}
$$

where constants $C_{i j k}^{\ell m n}$ are defined in (2.11).

We call the condition, $(\mathrm{N})_{1}$ and $(\mathrm{N})_{2}$, on the nonlinear term $F$ the null condition for nonlinear elastic waves. We will list typical nonlinear terms in $F$ satisfying the condition $(\mathrm{N})_{1}$ or $(\mathrm{N})_{2}$.

\section{Lemma 3.1}

(i) $Q_{\ell m}\left(\partial_{n} u^{j}, u^{k}\right)=\partial_{\ell} \partial_{n} u^{j} \partial_{m} u^{k}-\partial_{m} \partial_{n} u^{j} \partial_{\ell} u^{k}$ in $F^{i}$ satisfy the null condition. More precisely, $Q_{\ell m}\left(\partial_{n} u^{j}, u^{k}\right)$ satisfy

$$
\sum_{\ell m n} C_{i j k}^{\ell m n} X_{\ell} X_{m} X_{n}=0 \text { for any } i, j, k
$$

(ii) The components of $\partial_{n} u^{k} \partial_{\ell}$ rot $u$ and $\partial_{\ell} \partial_{m} u^{j}$ rot $u$ in $F^{i}$ satisfy the condition $(\mathrm{N})_{1}$, where rot $u=\nabla \wedge u$.

(iii) $\partial_{n} u^{k} \partial_{\ell} \operatorname{div} u, \partial_{\ell} \partial_{m} u^{j} \operatorname{div} u$ in $F^{i}$ and $F=\partial_{n} u^{k} \operatorname{grad}\left(\partial_{m} u^{j}\right)$ satisfy the condition $(N)_{2}$.

Proof. The assertions (i) and (ii) are easily verified by the definition of $C_{i j k}^{\ell m n}$. The polynomial corresponding to $\partial_{\ell} \partial_{m} u^{j} \operatorname{div} u$ in $F^{i}$ is

$$
X_{i} X_{j} X_{\ell} X_{m}(\xi \cdot X) \quad \text { for } \quad i \neq j
$$

or

$$
\left(|X|^{2}-X_{i}^{2}\right) X_{\ell} X_{m}(\xi \cdot X) \quad \text { for } \quad i=j
$$


The polynomials corresponding to $\partial_{n} u^{k} \partial_{\ell} \operatorname{div} u$ in $F^{i}$ and $\partial_{n} u^{k} \operatorname{grad}\left(\partial_{m} u^{j}\right)$ are

$$
X_{i} \xi_{k} X_{\ell} X_{n}\left\{\left(|X|^{2}-X_{i}^{2}\right)-\sum_{h \neq i} X_{h}^{2}\right\}
$$

and

$$
X_{j} \xi_{k} X_{m} X_{n}\left\{\left(|X|^{2}-X_{j}^{2}\right)-\sum_{h \neq j} X_{h}^{2}\right\}
$$

respectively. Therefore the assertion (iii) is proved.

We rewrite the nonlinear term $F\left(\nabla u, \nabla^{2} u\right)$ to be satisfied the null condition. We first show that the nonlinear term involving $\sigma_{2}$ and $\sigma_{3}$ is the sum of null forms of type (i) in Lemma 3.1.

Lemma 3.2. Let $Q_{1}(u, \nabla u)$ be the nonlinear term involving $\sigma_{2}$ and $\sigma_{3}$. Then the $i$-th component $Q_{1}^{i}(u, \nabla u)$ of $Q_{1}(u, \nabla u)$ can be expressed by

$$
\begin{aligned}
& 2\left(\sigma_{2}-\sigma_{3}\right) \sum_{j, k=1}^{3}\left\{2 Q_{j k}\left(\partial_{j} u^{i}, u^{k}\right)+Q_{j k}\left(\partial_{k} u^{j}, u^{i}\right)+Q_{i j}\left(\partial_{j} u^{k}, u^{k}\right)\right\} \\
& +2 \sigma_{3} \sum_{j, k=1}^{3}\left\{2 Q_{i j}\left(\partial_{k} u^{k}, u^{j}\right)+Q_{j i}\left(\partial_{k} u^{j}, u^{k}\right)\right\}
\end{aligned}
$$

Proof. It follows from (2.5) and (2.8) that

$$
\begin{aligned}
& Q_{1}(u, \nabla u) \\
= & 2\left(\sigma_{2}-\sigma_{3}\right) \operatorname{div}\left\{\operatorname{tr}\left(G^{t} G\right) I-{ }^{t} G G+2(\operatorname{tr} G) G-G^{2}-G^{t} G\right\} \\
& +2 \sigma_{3} \operatorname{div}\left\{2(\operatorname{tr} G)^{2} I-2(\operatorname{tr} G)^{t} G+\left({ }^{t} G\right)^{2}-\left(\operatorname{tr} G^{2}\right) I\right\} .
\end{aligned}
$$

Making use of identities

$$
\begin{aligned}
& \operatorname{div}\left(\operatorname{tr}\left(G^{t} G\right) I\right)^{i}=\partial_{i}|\nabla u|^{2}, \\
& \operatorname{div}\left({ }^{t} G G\right)^{i}=\frac{1}{2} \partial_{i}|\nabla u|^{2}+\partial_{i} u \cdot \Delta u \\
& \operatorname{div}((\operatorname{tr} G) G)^{i}=\operatorname{div} u \Delta u^{i}+\nabla \operatorname{div} u \cdot \nabla u^{i}, \\
& \operatorname{div}\left(G^{2}\right)^{i}=\Delta u \cdot \nabla u^{i}+\sum_{j, k} \partial_{j} \partial_{k} u^{i} \partial_{j} u^{k}, \\
& \operatorname{div}\left(G^{t} G\right)^{i}=\nabla \operatorname{div} u \cdot \nabla u^{i}+\sum_{j k} \partial_{j} \partial_{k} u^{i} \partial_{k} u^{j},
\end{aligned}
$$


we have

$$
\begin{aligned}
& \left.\operatorname{div}\left(\operatorname{tr}\left(G^{t} G\right) I\right) I-{ }^{t} G G\right)^{i}=\sum_{j, k} Q_{i j}\left(\partial_{j} u^{k}, u^{k}\right), \\
& \operatorname{div}\left(2(\operatorname{tr} G) G-G^{2}-G^{t} G\right) \\
= & 2\left(\operatorname{div} u \Delta u^{i}-\sum_{j k} \partial_{j} \partial_{k} u^{i} \partial_{j} u^{k}\right)+\left(\nabla \operatorname{div} u \cdot \nabla u^{i}-\Delta u \cdot \nabla u^{i}\right) \\
= & 2 \sum_{j k}\left\{Q_{j k}\left(\partial_{j} u^{i}, u^{k}\right)+Q_{j k}\left(\partial_{k} u^{j}, u^{i}\right)\right\}
\end{aligned}
$$

Moreover, making use of identities

$$
\begin{aligned}
& \operatorname{div}\left((\operatorname{tr} G)^{2} I\right)^{i}=\partial_{i}(\operatorname{div} u)^{2} \\
& \left.\operatorname{div}(\operatorname{tr} G)^{t} G\right)^{i}=\operatorname{div} u \partial_{i} \operatorname{div} u+\nabla \operatorname{div} u \cdot \partial_{i} u \\
& \operatorname{div}\left(\left({ }^{t} G\right)^{2}\right)^{i}=\nabla \operatorname{div} u \cdot \partial_{i} u+\sum_{j k} \partial_{j} \partial_{i} u^{k} \partial_{k} u^{j} \\
& \left.\operatorname{div}\left(\left(\operatorname{tr} G^{2}\right) I\right)^{i}=\partial_{i}\left(\sum_{j k} \partial_{j} u^{k} \partial_{k} u^{j}\right)\right)
\end{aligned}
$$

we have

$$
\begin{aligned}
& \left.2 \operatorname{div}\left((\operatorname{tr} G)^{2} I\right)-(\operatorname{tr} G)^{t} G\right)^{i}=2 \sum_{j, k} Q_{i j}\left(\partial_{k} u^{k}, u^{j}\right), \\
& \operatorname{div}\left(\left({ }^{t} G\right)^{2}-\left(\operatorname{tr} G^{2}\right) I\right)^{i}=\sum_{j k} Q_{j i}\left(\partial_{k} u^{j}, u^{k}\right) .
\end{aligned}
$$

Therefore, the relation (3.5) follows from (3.6), (3.9), (3.10), (3.14) and (3.15).

We find from (2.5), (3.7), (3.8), (3.11), (3.12), (3.13) and Lemma 3.2 that

$$
\begin{aligned}
& F^{i}\left(\nabla u, \nabla^{2} u\right)=4\left(\sigma_{111}+3 \sigma_{12}\right) \partial_{i}(\operatorname{div} u)^{2} \\
& +2\left(\sigma_{11}-\sigma_{12}\right)\left\{2\left(\operatorname{div} u \Delta u^{i}+\nabla \operatorname{div} u \cdot \nabla u^{i}\right)+\partial_{i}|\nabla u|^{2}\right\} \\
& -2 \sigma_{12}\left\{\partial_{i}(\operatorname{div} u)^{2}+2 \nabla \operatorname{div} \cdot \partial_{i} u+\sum_{j \cdot k} \partial_{i}\left(\partial_{j} u^{k} \partial_{k} u^{j}\right)\right\} \\
& +Q_{1}^{i}(u, \nabla u) \quad(i=1,2,3)
\end{aligned}
$$

Remark 3.1. Sideris [15] has imposed the condition $\sigma_{11}-\sigma_{12}=0$ and $8\left(\sigma_{111}+3 \sigma_{12}\right)-$ $12 \sigma_{12}=0$, having the coefficients of $4\left(\sigma_{111}+3 \sigma_{12}\right)$ and $-2 \sigma_{12}$ the same nonlinearity 
condition. Thus Sideris' null condition is

$$
\sigma_{11}-\sigma_{12}=0 \text { and } 2 \sigma_{111}+3 \sigma_{12}=0
$$

We next show that $F\left(\nabla u, \nabla^{2} u\right)$ can be written by using the terms div $u$ and rot $u$, except for the null forms of type (i) in Lemma 3.1.

Proposition 3.2.

$$
\begin{aligned}
& F\left(\nabla u, \nabla^{2} u\right)=2\left(2 \sigma_{111}+3 \sigma_{11}\right) \operatorname{grad}(\operatorname{div} u)^{2} \\
& +2\left(\sigma_{11}-\sigma_{12}\right)\left(\operatorname{grad}|\operatorname{rot} u|^{2}-2 \operatorname{rot}(\operatorname{div} u \operatorname{rot} u)\right) \\
& +Q(u, \nabla u)
\end{aligned}
$$

where $Q=Q_{1}+Q_{2}$ and

$$
Q_{2}^{i}(u, \partial u)=4\left(\sigma_{11}-2 \sigma_{12}\right) \sum_{j \cdot k}\left(Q_{i k}\left(u^{k}, \partial_{j} u^{j}\right)-Q_{j k}\left(u^{j}, \partial_{i} u^{k}\right)\right)
$$

Proof. The fundamental identities for vector fields

$$
\begin{aligned}
& \Delta u=\operatorname{grad} \operatorname{div} u-\operatorname{rot} \operatorname{rot} u, \\
& |\nabla u|^{2}=|\operatorname{rot} u|^{2}+\sum_{j \cdot k} \partial_{j} u^{k} \partial_{k} u^{j},
\end{aligned}
$$

$\nabla \operatorname{div} u \cdot \nabla u^{i}=-(\nabla \operatorname{div} u \wedge \operatorname{rot} u)^{i}+\nabla \operatorname{div} u \cdot \partial_{i} u$

yield

$$
\begin{aligned}
& 2\left(\operatorname{div} u \Delta u^{i}+\nabla \operatorname{div} u \cdot \nabla u^{i}\right)+\partial_{i}|\nabla u|^{2} \\
= & \partial_{i}|\operatorname{rot} u|^{2}-2 \operatorname{rot}(\operatorname{div} u \operatorname{rot} u)^{i}+\partial_{i}(\operatorname{div} u)^{2} \\
& +2 \nabla \operatorname{div} u \cdot \partial_{i} u+\partial_{i} \sum_{j k} \partial_{j} u^{k} \partial_{k} u^{j}
\end{aligned}
$$

It then follows from (3.16) and (3.19) that

$$
\begin{aligned}
& F^{i}\left(\nabla u, \nabla^{2} u\right)=2\left(2 \sigma_{111}+\sigma_{11}+4 \sigma_{12}\right) \partial_{i}(\operatorname{div} u)^{2} \\
& +2\left(\sigma_{11}-\sigma_{12}\right)\left\{\left(\partial_{i}|\operatorname{rot} u|^{2}-2 \operatorname{rot}(\operatorname{div} u \operatorname{rot} u)^{i}\right\}\right. \\
& +2\left(\sigma_{11}-2 \sigma_{12}\right)\left(2 \nabla \operatorname{div} u \cdot \partial_{i} u+\partial_{i} \sum_{j k} \partial_{j} u^{k} \partial_{k} u^{j}\right) \\
& +Q_{1}(u, \nabla u) .
\end{aligned}
$$


Making use of indentities

$$
\begin{aligned}
& \nabla \operatorname{div} u \cdot \partial_{i} u=\operatorname{div} u \partial_{i} \operatorname{div} u-\sum_{j k} Q_{i k}\left(\partial_{j} u^{j}, u^{k}\right), \\
& \partial_{i} \sum_{j, k} \partial_{j} u^{k} \partial_{k} u^{j}=\partial_{i}(\operatorname{div} u)^{2}+2 \sum_{j k} Q_{j k}\left(\partial_{i} u^{k}, u^{j}\right),
\end{aligned}
$$

we conclude combining (3.18) and (3.20) that Proposition 3.2 is valid.

Now we made ready to state the first aim of this paper.

Theorem 3.1. The nonlinear term $F\left(\nabla u, \nabla^{2} u\right)$ satisfies the null condition if and only if

$$
2 \sigma_{111}+3 \sigma_{11}=0
$$

Proof. We find from Lemma 3.1 that the nonlinear terms in (3.17) except for grad $(\operatorname{div} u)^{2}$ satisfy the null condition. Note that the condition $(\mathrm{N})_{1}$ implies $C_{i i i}^{i i i}=0$ for $i=1,2,3$. Since the coefficient of $\partial_{i} u^{i} \partial_{i}^{2} u^{i}$ in $F_{i}$ corresponds injectively to the one of $X_{i}^{6}$, it follows from the condition $(\mathrm{N})_{1}$ that $2 \sigma_{111}+3 \sigma_{11}=0$.

Remark 3.2. John [9] has proved that if $2 \sigma_{111}+3 \sigma_{11} \neq 0$ then spherically symmetric solutions of (2.8) with small data blow up.

Remark 3.3. The nonlinear term $\operatorname{grad}(\operatorname{div} u)^{2}$ satisfies the energy symmetry condition (2.12) in Proposition 2.1.

\section{Global existence theorem}

Assume that the nonlinear term $F\left(\nabla u, \nabla^{2} u\right)$ satisfies the null condition. Then, from (2.8), Proposition 3.2 and Theorem 3.1, we can formulate the initial value problem for nonlinear elastic waves:

$$
\begin{aligned}
& \partial_{t}^{2} u-c_{2}^{2} \Delta u-\left(c_{1}^{2}-c_{2}^{2}\right) \operatorname{grad} \operatorname{div} u \\
= & 2\left(\sigma_{11}-\sigma_{12}\right)\left(\operatorname{grad}|\operatorname{rot} u|^{2}-2 \operatorname{rot}(\operatorname{div} u \operatorname{rot} u)\right)+Q(u, \nabla u), \\
& u(0, x)=\varepsilon f(x), \partial_{t} u(0, x)=\varepsilon g(x),
\end{aligned}
$$


where $f, g \in C_{0}^{\infty}\left(\mathbb{R}^{3}\right)$ and $\varepsilon$ is small positive parameter.

The second aim of this paper is to prove the following

Theorem 4.1. There exists a positive constant $\varepsilon_{0}$ such that the initial value problem (4.1) has a unique global in time $C^{\infty}$-solution $u$ for any $\varepsilon\left(0<\varepsilon \leq \varepsilon_{0}\right)$.

\section{Notations and commutation relations}

The space-time gradient will be denoted by

$$
\partial=\left(\partial_{0}, \partial_{1}, \partial_{2}, \partial_{3}\right)=\left(\partial_{0}, \nabla\right)
$$

where

$$
\partial_{0}=\partial_{t}=\frac{\partial}{\partial t}, \quad \partial_{i}=\frac{\partial}{\partial x_{i}} \quad(i=1,2,3)
$$

The angular momentum operators are the vector fields

$$
\Omega=\left(\Omega_{1}, \Omega_{2}, \Omega_{3}\right)=x \wedge \nabla
$$

Then the spatial derivatives can be decomposed into radial and angular components

$$
\nabla=\frac{x}{r} \partial_{r}-\frac{x}{r^{2}} \wedge \Omega, \quad \text { where } r=|x|, \partial_{r}=\frac{x}{r} \cdot \nabla
$$

We also use the vector fields

$$
\tilde{\Omega}=\Omega I+U
$$

where

$$
U^{1}=\left(\begin{array}{ccc}
0 & 0 & 0 \\
0 & 0 & 1 \\
0 & -1 & 0
\end{array}\right), U^{2}=\left(\begin{array}{ccc}
0 & 0 & -1 \\
0 & 0 & 0 \\
1 & 0 & 0
\end{array}\right), U^{3}=\left(\begin{array}{ccc}
0 & 1 & 0 \\
-1 & 0 & 0 \\
0 & 0 & 0
\end{array}\right)
$$

The seven vector fields will be written as

$$
\begin{aligned}
& \Gamma=\left(\Gamma_{0}, \cdots, \Gamma_{6}\right)=(\partial, \Omega), \\
& \tilde{\Gamma}=\left(\tilde{\Gamma}_{0}, \cdots, \tilde{\Gamma}_{6}\right)=(\partial I, \tilde{\Omega}) .
\end{aligned}
$$


The commutator of any two $\Gamma^{\prime} s$ is either 0 or $\nabla$. By $\Gamma^{a},|a|=k$, will be meant an ordered product of $k$ vector fields $\Gamma_{a_{1}} \cdots \Gamma_{a_{k}}$.

The linear hyperbolic operator $L$ in (2.7) commutes with any $\tilde{\Gamma}$. Moreover, wave operators $\partial_{t}^{2}-c_{i}^{2} \Delta(i=1,2)$ also commute with any $\Gamma$. The new commutation relations

$$
\tilde{\Omega} \operatorname{grad} f=\operatorname{grad} \Omega f, \operatorname{div} \tilde{\Omega} u=\Omega \operatorname{div} u
$$

for any scaler $f$ and vector field $u$ play a crucial role for handling the nonlinear term $\operatorname{grad}|\operatorname{rot} u|^{2}$.

Applying div and rot to the equation (4.1), we get

$$
\begin{aligned}
& \partial_{t}^{2} \operatorname{div} u-c_{1}^{2} \Delta \operatorname{div} u \\
= & 2\left(\sigma_{11}-\sigma_{12}\right) \Delta|\operatorname{rot} u|^{2}+\operatorname{div} Q(u, \nabla u),
\end{aligned}
$$

$$
\begin{aligned}
& \partial_{t}^{2} \operatorname{rot} u-c_{2}^{2} \Delta \operatorname{rot} u \\
= & -4\left(\sigma_{11}-\sigma_{12}\right)(\operatorname{rot})^{2}(\operatorname{div} u \operatorname{rot} u)+\operatorname{rot} Q((u, \nabla u),
\end{aligned}
$$

In order to weighted $L^{\infty}-L^{2}$ estimates for the solutions $u$, div $u$ and $\operatorname{rot} u$ of (4.1), (5.3) and (5.4), respectively, we adopt the following weight functions

$$
\begin{aligned}
& w_{i}(t, r)=(1+r)\left(1+\left|c_{i} t-r\right|\right) \quad(i=1,2), \\
& w(t, r)=\min _{i=1,2} w_{i}(t, r), \quad r=|x| .
\end{aligned}
$$

We also use the following norms for a vector field $u$

$$
\begin{aligned}
& {[\nabla u]_{k, t}=\sum_{|a| \leq k} \sup _{0 \leq s \leq t} \sup _{x \in \mathbb{R}^{3}}\left\{\sum_{i=1}^{3}\left|w(s,|x|) \Gamma^{a} \partial_{i} u(s, x)\right|\right.} \\
& \|\nabla u(s)\|_{k}=\sum_{|a| \leq k} \sum_{i=1}^{3}\left\|\Gamma^{a} \partial_{i} u(s, \cdot)\right\|_{L^{2}\left(\mathbb{R}^{3}\right)} \\
& \|\partial u(s)\|_{k}=\sum_{|a| \leq k} \sum_{\alpha=0}^{3}\left\|\Gamma^{a} \partial_{\alpha} u(s, \cdot)\right\|_{L^{2}\left(\mathbb{R}^{3}\right)} \\
& \|u\|_{k, t}=\sup _{0 \leq s \leq t}\|u(s)\|_{k} .
\end{aligned}
$$


Here $|v|$ stands for the norm of $v \in \mathbb{R}^{d}(d \geq 1)$.

6. Weighted $L^{\infty}-L^{\infty}$ estimates

In order to establish weighted $L^{\infty}-L^{\infty}$ estimates for the solution $u$ of (4.1), we make use of precise expressions of solution to the homogeneous linear problem

$$
\begin{aligned}
& L v=\partial_{t}^{2} v-c_{2}^{2} \Delta v-\left(c_{1}^{2}-c_{2}^{2}\right) \operatorname{grad} \operatorname{div} v=0 \\
& v(0, x)=0, \partial_{t} v(0, x)=g(x)
\end{aligned}
$$

Proposition 6.1. The solution $v$ of $(6.1)$ is expressed in two manner:

$$
\begin{aligned}
& v^{i}(t, x)=\frac{t}{4 \pi} \int_{|\omega|=1} g^{i}\left(x+c_{2} t \omega\right) d S_{\omega} \\
& +\frac{t}{4 \pi} \sum_{j=1}^{2}(-1)^{j-1} \int_{|\omega|=1} \omega_{i} \sum_{k=1}^{3} \omega_{k} g^{k}\left(x+c_{j} t \omega\right) d S_{\omega} \\
& -\frac{t}{4 \pi} \int_{c_{2} t}^{c_{1} t} \tau^{-1} d \tau \int_{|\omega|=1} \sum_{k=1}^{3}\left(\delta_{i k}-3 \omega_{i} \omega_{k}\right) g^{k}(x+\tau \omega) d S_{\omega}
\end{aligned}
$$

and

$$
\begin{aligned}
v(t, x)= & \frac{t}{4 \pi} \int_{|\omega|=1} g\left(x+c_{1} t \omega\right) d S_{\omega} \\
& +\frac{t}{4 \pi} \int_{c_{2} t \leq|y| \leq c_{1} t}|y|^{-3} y \wedge(\operatorname{rot} g)(x+y) d y
\end{aligned}
$$

The expression (6.2) is standard (for instance see [10]). The new expression (6.3) will be used to get a good decay of the nonlinear term $\operatorname{grad}|\operatorname{rot} u|^{2}$. Here we will prove first (6.3) and then (6.2) for the completeness.

Proof of Proposition 6.1. We observe that each component $v^{i}$ of the solution $v$ satisfies the scaler equation of fourth order

$$
\left(\partial_{t}^{2}-c_{2}^{2} \Delta\right)\left(\partial_{t}^{2}-c_{1}^{2} \Delta\right) v^{i}=0
$$


which can be solved successively first for $\left(\partial_{t}^{2}-c_{1}^{2} \Delta\right) v^{i}$ and then for $v^{i}$. The solution $w^{i}$ of the wave equation

$$
\partial_{t}^{2} w^{i}-c_{2}^{2} \Delta w^{i}=0
$$

with initial values

$$
w^{i}(0, x)=0, \partial_{t} w^{i}(0, x)=\left(c_{1}^{2}-c_{2}^{2}\right)\left(\partial_{i} \operatorname{div} g-\Delta g^{i}\right)(x)
$$

is explicitly expressed by

$$
w^{i}(t, x)=\frac{c_{1}^{2}-c_{2}^{2}}{4 \pi} \int_{|\omega|=1} t\left(\partial_{i} \operatorname{div} g-\Delta g^{i}\right)\left(x+c_{2} t \omega\right) d S_{\omega}
$$

Thus we find that the solution $v$ of $(6.1)$ is expressed by

$$
\begin{aligned}
& v(t, x)=\frac{t}{4 \pi} \int_{|\omega|=1} g\left(x+c_{1} t \omega\right) d S_{\omega}+\frac{c_{1}^{2}-c_{2}^{2}}{(4 \pi)^{2}} \int_{0}^{t}(t-s) d s \times \\
& \times \int_{|\zeta|=1} \int_{|\omega|=1} s h\left(x+c_{1}(t-s) \zeta+c_{2} s \omega\right) d S_{\omega} d S_{\zeta},
\end{aligned}
$$

where

$$
h=\operatorname{grad} \operatorname{div} g-\Delta g .
$$

We make use of the fundamental identity for iterated spherical means

$$
\begin{aligned}
& \frac{1}{(4 \pi)^{2}} \int_{|\zeta|=1} \int_{|\omega|=1} \varphi(x+r \zeta+\rho \omega) d S_{\omega} d S_{\zeta} \\
= & \frac{1}{8 \pi r \rho} \int_{r-\rho}^{r+\rho} \lambda d \lambda \int_{|\omega|=1} \varphi(x+\lambda \omega) d S_{\omega}
\end{aligned}
$$

(for instance, see [5]). Then, it follows from (6.4) and (6.5) that

$$
\begin{aligned}
& v(t, x)=\frac{t}{4 \pi} \int_{|\omega|=1} g\left(x+c_{1} t \omega\right) d S_{\omega} \\
& +\frac{c_{1}^{2}-c_{2}^{2}}{8 \pi c_{1} c_{2}} \int_{0}^{t} d s \int_{\left|c_{1}(t-s)-c_{2} s\right|}^{c_{1}(t-s)+c_{2} s} \lambda d \lambda \int_{|\omega|=1} h(x+\lambda \omega) d S_{\omega} .
\end{aligned}
$$


Denote the second integral in the right hand side of (6.6) by $J(t, x)$. Then, inverting the order of the $(s, \lambda)$ integral yields

$$
\begin{aligned}
J(t, x)= & \frac{c_{1}-c_{2}}{4 \pi c_{1} c_{2}} \int_{0}^{c_{2} t} \lambda^{2} d \lambda \int_{|\omega|=1}(\operatorname{grad} \operatorname{div} g-\Delta g)(x+\lambda \omega) d S_{\omega} \\
& +\frac{1}{4 \pi c_{1}} \int_{c_{2} t}^{c_{1} t}\left(-\lambda^{2}+c_{1} t \lambda\right) d \lambda \int_{|\omega|=1}(\operatorname{grad} \operatorname{div} g-\Delta g)(x+\lambda \omega) d S_{\omega} \\
= & \frac{c_{1}-c_{2}}{4 \pi c_{1} c_{2}} \int_{0}^{c_{2} t}(\operatorname{grad} \operatorname{div} g-\Delta g)(x+y) d y \\
& -\frac{1}{4 \pi c_{1}} \int_{c_{2} t}^{c_{1} t}(\operatorname{grad} \operatorname{div} g-\Delta g)(x+y) d y \\
& +\frac{t}{4 \pi} \int_{c_{2} t \leq|y| \leq c_{1} t} \frac{(\operatorname{grad} \operatorname{div} g-\Delta g)(x+y)}{|y|} d y .
\end{aligned}
$$

Making use of the divergence theorem, we have

$$
\begin{aligned}
J(t, x)= & \frac{1}{4 \pi c_{2}} \int_{|y|=c_{2} t} \frac{1}{c_{2} t} y \wedge \operatorname{rot} g(x+y) d S_{y} \\
& -\frac{1}{4 \pi c_{1}} \int_{|y|=c_{1} t} \frac{1}{c_{1} t} y \wedge \operatorname{rot} g(x+y) d S_{y} \\
& +\frac{t}{4 \pi} \int_{c_{2} t \leq|y| \leq c_{1} t} \frac{(\operatorname{grad} \operatorname{div} g-\Delta g)(x+y)}{|y|} d y .
\end{aligned}
$$

Since

$$
\frac{\partial_{i} \operatorname{div} g-\Delta g^{i}}{|y|}=\operatorname{div}\left(\frac{\partial_{i} g-\operatorname{grad} g^{i}}{|y|}\right)+\frac{1}{|y|^{3}} \sum_{k} y_{k}\left(\partial_{i} g^{k}-\partial_{k} g^{i}\right)
$$

it follows from (6.7) and the divergence theorem that

$$
J(t, x)=\frac{t}{4 \pi} \int_{c_{2} t \leq|y|<c_{1} t}|y|^{-3} y \wedge \operatorname{rot} g(x+y) d y
$$

which implies (6.3).

On the other hand, it holds that

$$
\begin{aligned}
& |y|^{-3} \sum_{k} y_{k}\left(\partial_{i} g^{k}-\partial_{k} g^{i}\right) \\
= & \sum_{k}\left\{\partial_{i}\left(|y|^{-3} y_{k} g^{k}\right)-\partial_{k}\left(|y|^{-3} y_{k} g^{i}\right)-g^{k}\left(\delta_{i k}|y|^{-3}-3|y|^{-5} y_{i} y_{k}\right)\right\}
\end{aligned}
$$


Integration by parts yields

$$
\frac{t}{4 \pi} \int_{c_{2} t \leq y \leq c_{1} t} \sum_{k}\left\{\partial_{i}\left(|y|^{-3} y_{k} g^{k}\right)-\partial_{k}\left(|y|^{-3} y_{k} g^{i}\right)\right\} d y
$$

$$
\begin{aligned}
= & \frac{t}{4 \pi} \int_{|\omega|=1} \omega_{i} \sum_{k} \omega_{k} g^{k}\left(x+c_{1} t \omega\right) d S_{\omega}-\frac{t}{4 \pi} \int_{|\omega|=1} g^{i}\left(x+c_{1} t \omega\right) d S_{\omega} \\
& -\frac{t}{4 \pi} \int_{|\omega|=1} \omega_{i} \sum_{k} \omega_{k} g^{k}\left(x+c_{2} t \omega\right) d S_{\omega}+\frac{t}{4 \pi} \int_{|\omega|=1} g^{i}\left(x+c_{2} t \omega\right) d S_{\omega} .
\end{aligned}
$$

Therefore, the assertion (6.2) follows from (6.6)-(6.10).

Let $u$ be the solution of the problem

$$
\begin{aligned}
& L u(t, x)=F(t, x), \\
& u(0, x)=\partial_{t} u(0, x)=0 .
\end{aligned}
$$

Then, by the standard expression (6.2) and the Duhamel's principle, we get

$$
u^{i}(t, x)=\frac{1}{4 \pi} \int_{0}^{t}(t-s) d s \int_{|\omega|=1} F^{i}\left(s, x+c_{2}(t-s) \omega\right) d S_{\omega}
$$

$$
\begin{aligned}
& +\frac{1}{4 \pi} \sum_{j=1}^{2}(-1)^{j-1} \int_{0}^{t}(t-s) d s \int_{|\omega|=1} \omega_{i} \sum_{k=1}^{3} \omega_{k} F^{k}\left(s, x+c_{j}(t-s) \omega\right) d S_{\omega} \\
& -\frac{1}{4 \pi} \int_{0}^{t}(t-s) d s \int_{c_{2}(t-s)}^{c_{1}(t-s)} \tau^{-1} d \tau \int_{|\omega|=1} \sum_{k=1}^{3}\left(\delta_{i k}-3 \omega_{i} \omega_{k}\right) F^{k}(x+\tau \omega) d S_{\omega}
\end{aligned}
$$

Let $I$ be the integral operator defined by (6.12). Then we have

$$
I(F)(t, x)=u(t, x), I(\nabla F)(t, x)=\nabla u(t, x) .
$$

To describe weighted $L^{\infty}-L^{\infty}$ estimates, we introduce some notations

$$
\begin{aligned}
& z_{\mu, \nu}^{(j)}(s, \lambda)=\left(1+\left|c_{j} s-\lambda\right|\right)^{\mu}(1+s+\lambda)^{\nu}, c_{0}=0, \\
& M_{\mu, \nu, k}^{(j)}(\varphi)=\sum_{|a| \leq k} \sup _{0 \leq s \leq t} \sup _{y \in \mathbb{R}^{3}}|y| z_{\mu, \nu}^{(j)}(s,|y|)\left|\Gamma^{a} \varphi(s, y)\right| \\
& \quad(j=0,1,2) .
\end{aligned}
$$


Here $\varphi$ is a scaler or vector valued function.

The following proposition will be proved in Section 10 .

Proposition 6.2. It holds for some positive constant $C$ depending on $c_{1}, c_{2}$ and $\mu$ that

$$
\begin{aligned}
& |I(F)(t, x)| \leq C(1+t+|x|)^{-1}(\log (2+t))^{2} M_{1,1,0}^{(j)}(F), \\
& |I(\partial F)(t, x)| \leq C w(t, x)^{-1}(\log (2+t))^{2} M_{1,1,1}^{(j)}(F), \\
& |I(\partial F)(t, x)| \leq C w(t, x)^{-1} M_{\mu, \mu, 1}^{(j)}(F), \\
& \quad(j=0,1,2, \mu>1),
\end{aligned}
$$

where the weighted function $w(t, r)$ is defined in (5.5).

Let $u_{i}$ be the solution of the problem

$$
\begin{aligned}
& \partial_{t}^{2} v_{i}-c_{i}^{2} \Delta v_{i}=h(t, x) \\
& v_{i}(0, x)=\partial_{t} v_{i}(0, x)=0 .
\end{aligned}
$$

Then, it is known that

$$
v_{i}(t, x)=\frac{1}{4 \pi} \int_{0}^{t}(t-s) d s \int_{|\omega|=1} h\left(s, x+c_{i}(t-s) \omega\right) d S_{\omega}
$$

Let $I_{i}, i=1,2$, be the integral operator defined by (6.15). Then we have

$$
I_{i}(h)(t, x)=v_{i}(t, x), I_{i}(\nabla h)(t, x)=\nabla v_{i}(t, x) .
$$

Moreover, the new expression (6.3) and the Duhamel's principle give an important formula

$$
I(\operatorname{grad} \varphi)(t, x)=I_{1}(\operatorname{grad} \varphi)(t, x)
$$

To obtain the weighted $L^{\infty}$ estimates for the solutions div $u, \operatorname{rot} u$ of (5.3), (5.4) and the nonlinear term grad $|\operatorname{rot} u|^{2}$, we will use the results of Proposition 3.1 in [16]. 
Proposition 6.3. It holds for some positive constant $C$ depending on $c_{1}, c_{2}$ and $\mu$ that

$$
\begin{aligned}
& \left|I_{i}(\partial \varphi)(t, x)\right| \leq C w_{i}(t,|x|)^{-1} \log (2+t) M_{1,1,1}^{(j)}(\varphi) \\
& \left|I_{i}(\partial \varphi)(t, x)\right| \leq C w_{i}(t,|x|)^{-1} M_{\mu, \mu, 1}^{(j)}(\varphi) \\
& \quad \text { for } j=0,1,2 \text { and } \mu>1
\end{aligned}
$$

and

(iii)

$$
\begin{aligned}
& \left|I_{i}(\partial \varphi)(t, x)\right| \leq C(1+|x|)^{-1}\left(1+\left|c_{i} t-\right| x||\right)^{-\nu} M_{\mu, \nu, 1}^{(j)}(\varphi) \\
& \quad \text { for } \nu>0, \mu>1 \text { and } j \neq i .
\end{aligned}
$$

\section{Weighted $L^{\infty}-L^{2}$ estimates}

In this Section, we will establish the $L^{\infty}-L^{2}$ estimates for the solution $u$ of (4.1).

Proposition 7.1. Let $u$ be the solution to the initial value problem (4.1). Then there exists a positive constant $C_{N}^{\prime}$ depending on $N$, initial values $f, g$ and propagation speeds $c_{1}, c_{2}$ such that

$$
[\nabla u]_{N, t} \leq C_{N}^{\prime}\left(\varepsilon+\|\nabla u\|_{N+7}^{4}\right)
$$

provided $\varepsilon<1$ and $[\nabla u]_{[(N+5) / 2], t}<1$.

Proof. Let $u_{0}$ be the solution of the homogeneous equation

$$
\begin{aligned}
& L u_{0}=\partial_{t}^{2} u_{0}-c_{2}^{2} \Delta u_{0}-\left(c_{1}^{2}-c_{2}^{2}\right) \operatorname{grad} \operatorname{div} u_{0}=0, \\
& u_{0}(0, x)=\varepsilon f(x), \partial_{t} u_{0}(0, x)=\varepsilon g(x) .
\end{aligned}
$$

Since $L$ commutes with $\tilde{\Gamma}$, we find successively from Theorem 1 of [10] that

$$
\left|\Gamma^{a} u_{0}(t, x)\right| \leq C_{N} \varepsilon w(t,|x|)^{-1} \text { for }|a| \leq N
$$


Applying div and rot to (7.2), we have

$$
\begin{aligned}
& \partial_{t}^{2} \operatorname{div} u_{0}-c_{1}^{2} \Delta \operatorname{div} u_{0}=0 \\
& \operatorname{div} u_{0}(0, x)=\varepsilon \operatorname{div} f(x), \partial_{t} \operatorname{div} u_{0}(0, x)=\varepsilon \operatorname{div} g(x) .
\end{aligned}
$$

and

$$
\begin{aligned}
& \partial_{t}^{2} \operatorname{rot} u_{0}-c_{2}^{2} \Delta \operatorname{rot} u_{0}=0 \\
& \operatorname{rot} u_{0}(0, x)=\varepsilon \operatorname{rot} f(x), \partial_{t} \operatorname{rot} u_{0}(0, x)=\varepsilon \operatorname{rot} g(x)
\end{aligned}
$$

Since wave operators $\partial_{t}^{2}-c_{i}^{2} \Delta, i=1,2$, commutes with $\Gamma$, it follows from Theorem 1 (i) of $[12]$ that

$$
\begin{aligned}
& \left|\Gamma^{a} \operatorname{div} u_{0}(t, x)\right| \leq C_{N} \varepsilon w_{1}(t,|x|)^{-1} \\
& \quad \text { for }|a| \leq N . \\
& \left|\Gamma^{a} \operatorname{rot} u_{0}(t, x)\right| \leq C_{N} \varepsilon w_{2}(t,|x|)^{-1}
\end{aligned}
$$

Set

$$
u_{1}=u-u_{0}
$$

and apply $\tilde{\Gamma}^{a}$ to (7.7). Then, $\tilde{\Gamma}^{a} u_{1}$ satisfy the equation in the form

$$
\begin{aligned}
& L \tilde{\Gamma}^{a} u_{1}=\sum_{|b| \leq|a|} C_{a b} \Gamma^{b} F \\
& \tilde{\Gamma}^{a} u_{1}(0, x)=\partial_{t} \tilde{\Gamma}^{a} u_{1}(0, x)=0 .
\end{aligned}
$$

Here the nonlinear term of (4.1) is denoted again by $F=F\left(\nabla u, \nabla^{2} u\right)$. We define a weighted function $z(s, \lambda)$ by

$$
z(s, \lambda)^{-1}=\sum_{j=0}^{2} z_{1,1}^{(j)}(s, \lambda)^{-1}
$$

Note that $I\left(\partial_{t} F\right)=\partial_{t} u$, because $F(0, x)=0$. Then it follows from (6.13), (7.8), (7.9) and Proposition 6.2 (i)(ii) that

$$
\begin{aligned}
& \left|\tilde{\Gamma}^{a} u_{1}(t, x)\right| \leq C_{N}(1+t+|x|)^{-1}(\log (2+t))^{2} M_{N}(F), \\
& \left|\partial \tilde{\Gamma}^{a} u_{1}(t, x)\right| \leq C_{N} w(t,|x|)(\log (2+t))^{2} M_{N+1}(F)
\end{aligned}
$$


for $|a| \leq N$ where

$$
M_{k}(F)=\sum_{|a| \leq k} \sup _{0 \leq s \leq t} \sup _{y \in \mathbb{R}^{3}}|y| z(s,|y|)\left|\Gamma^{a} F\left(\nabla u, \nabla^{2} u\right)(s, y)\right| .
$$

Making use of Sobolev inequality (see [14])

$$
|y||f(y)| \leq C\left(\sum_{|a| \leq 2}\left\|\Omega^{a} f\right\|_{L^{2}\left(\mathbb{R}^{3}\right)}+\sum_{|a| \leq 1}\left\|\partial_{r} \Omega^{a} f\right\|_{L^{2}\left(\mathbb{R}^{3}\right)}\right)
$$

and the fact that $z(s,|y|) \leq C w(s,|y|)$, we have

$$
|y| z(s,|y|)\left|\nabla \Gamma^{b} u^{i}(s, y)\right|\left|\nabla \Gamma^{c} u^{i}(s, y)\right| \leq C_{k}[\nabla u]_{[k / 2], t}|| \nabla u \|_{k+2, t}
$$

for $|b|+|c| \leq k, 0 \leq s<t$, which implies

$$
M_{k}(F) \leq C_{k}[\nabla u]_{[(k+1) / 2], t}\|\nabla u\|_{k+3, t}
$$

Thus, we find successively from (7.3), (7.7), (7.10) and (7.11) that, for $|a| \leq N$,

$$
\begin{aligned}
& \left|\partial \Gamma^{a} u(t, x)\right| \\
\leq & C_{N} w(t,|x|)^{-1}(\log (2+t))^{2}\left(\varepsilon+[\nabla u]_{[(N+2) / 2], t}\|\nabla u\|_{N+4, t}\right) .
\end{aligned}
$$

Since $\Gamma^{a} \operatorname{div} F$ and $\Gamma^{a}$ rot $F$ can be written by a linear combination of terms

$$
\nabla \Gamma^{b} F^{i} \text { for }|b| \leq|a| \text { and } i=1,2,3 \text {, }
$$

we find from (5.3), (5.4), (7.4), (7.5) and (7.7) that

$$
\begin{aligned}
& \partial_{t}^{2} \Gamma^{a} \operatorname{div} u_{1}-c_{1}^{2} \Delta \Gamma^{a} \operatorname{div} u_{1}=\sum_{|b| \leq|a|} C_{a b} \nabla \Gamma^{b} F_{1} \\
& F_{1}=2\left(\sigma_{11}-\sigma_{12}\right) \operatorname{grad}|\operatorname{rot} u|^{2}+Q(u, \nabla u) \\
& \Gamma^{a} \operatorname{div} u_{1}(0, x)=\partial_{t} \Gamma^{a} \operatorname{div} u_{1}(0, x)=0
\end{aligned}
$$


and

$$
\begin{aligned}
& \partial_{t}^{2} \Gamma^{a} \operatorname{rot} u-c_{2}^{2} \Delta \Gamma^{a} \operatorname{rot} u=\sum_{|b| \leq|a|} C_{a b} \nabla \Gamma^{b} F_{2} \\
& F_{2}=-4\left(\sigma_{11}-\sigma_{12}\right) \operatorname{rot}(\operatorname{div} u \operatorname{rot} u)+Q(u, \nabla u) \\
& \Gamma^{a} \operatorname{rot} u(0, x)=\partial_{t} \Gamma^{a} \operatorname{rot} u(0, x)=0 .
\end{aligned}
$$

Hence, it follows from (6.16), (7.14), (7.15) and Proposition 6.3 (i) that

$$
\begin{aligned}
& \left|\Gamma^{a} \operatorname{div} u_{1}(t, x)\right| \leq C_{N} w_{1}(t,|x|)^{-1} \log (2+t) M_{N+1}\left(F_{1}\right) \\
& \left|\Gamma^{a} \operatorname{rot} u_{1}(t, x)\right| \leq C_{N} w_{2}(t,|x|)^{-1} \log (2+t) M_{N+1}\left(F_{2}\right) .
\end{aligned}
$$

Therefore, by the same argument to obtain (7.13), we find from (7.6), (7.7) and (7.16) that

$$
\begin{aligned}
& \left|\Gamma^{a} \operatorname{div} u(t, x)\right| \\
& \leq C_{N} w_{1}(t, x)^{-1} \log (2+t)\left(\varepsilon+[\nabla u]_{[(N+2) / 2], t}\|\nabla u\|_{N+4, t}\right), \\
& \left|\Gamma^{a} \operatorname{rot} u(t, x)\right| \\
& \leq C_{N} w_{2}(t, x)^{-1} \log (2+t)\left(\varepsilon+[\nabla u]_{[(N+2) / 2], t}\|\nabla u\|_{N+4, t}\right),
\end{aligned}
$$

In order to remove log terms from the inequalities above, it is necessary to further analyze the nonlinear terms. The following pointwise estimate follows from (5.1).

$$
\left|Q_{\ell m}\left(\partial_{n} u^{i}, u^{k}\right)\right| \leq C r^{-1}\left(\left|\Omega \nabla u^{i}\right|\left|\nabla u^{k}\right|+\left|\nabla^{2} u\right|\left|\Omega u^{k}\right|\right)
$$

Let $\Lambda_{i}$ be a conic neighbourhood of $i$-th characteristic $c_{i} t=|x|$ such that $\Lambda_{1} \cap \Lambda_{2}=\emptyset$. For instance, set

$$
\begin{aligned}
& \Lambda_{1}=\left\{(t, x) ; \frac{1}{2}|x|<c_{1} t<\frac{1}{3}\left(2+\frac{c_{1}}{c_{2}}\right)|x|\right\}, \\
& \Lambda_{2}=\left\{(t, x) ; \frac{1}{3}\left(2+\frac{c_{2}}{c_{1}}\right)|x|<c_{2} t<2|x|\right\} .
\end{aligned}
$$

Then we find from (7.12), (7.13), (7.19) and Lemma 2.1 of [13] that, for $(t, x) \in \Lambda_{1} \cup \Lambda_{2}$,

$$
\begin{aligned}
& \left|\Gamma^{a} Q(u, \nabla u)\right| \\
\leq & C_{N}(1+t+|x|)^{-3}\left\{\min _{i=1,2}\left(1+\left|c_{i} t-\right| x||\right)\right\}^{-1}(\log (2+t))^{4} \times \\
& \times\left(\varepsilon+[\nabla u]_{[(N+3) / 2], t}\|\nabla u\|_{N+5, t}^{2}\right)
\end{aligned}
$$


provided $\varepsilon<1$ and $[\nabla u]_{\{(N+3) / 2], t}<1$. Note that $\left\{\min _{i=1,2}\left(1+\left|c_{i} t-\right| x||\right)\right\}^{-1}$ is equivalent to

$$
\frac{1}{1+\left|c_{1} t-\right| x||}+\frac{1}{1+\left|c_{2} t-\right| x||}
$$

Then, for $(t, x) \in\left(\Lambda_{1} \cup \Lambda_{2}\right)^{c}$, the estimate (7.13) yields

$$
\begin{aligned}
& \left|\Gamma^{a} Q(u, \nabla u)\right| \leq C_{N} \sum_{|b|+|c| \leq N+1}\left|\nabla \Gamma^{b} u\right|\left|\nabla \Gamma^{c} u\right| \\
& \leq C_{N}(1+|x|)^{-2}(1+t+|x|)^{-2}(\log (2+t))^{4} \times \\
& \times\left(\varepsilon+\left.[\nabla u]_{[(N+3) / 2], t}|| \nabla u\right|_{N+5, t} ^{2}\right) .
\end{aligned}
$$

Moreover, the estimates (7.17) and (7.18) yield

$$
\begin{aligned}
& \left|\Gamma^{a} \operatorname{rot}(\operatorname{div} u \operatorname{rot} u)\right| \\
& \leq C_{N} w_{1}(t,|x|)^{-1} w_{2}(t,|x|)^{-1}(\log (2+t))^{2} \times \\
& \times\left(\varepsilon+\left.[\nabla u]_{[(N+3) / 2], t}|| \nabla u\right|_{N+5, t} ^{2}\right)
\end{aligned}
$$

Note that

$$
\begin{aligned}
& w_{1}(t, x) w_{2}(t, x) \\
& \geq C \begin{cases}(1+t+|x|)^{3}\left(1+\left|c_{i} t-\right| x||\right) & \text { for }(t, x) \in \Lambda_{i} \\
(1+|x|)^{2}(1+t+|x|)^{2} & \text { for }(t, x) \in\left(\Lambda_{1} \cup \Lambda_{2}\right)^{c} .\end{cases}
\end{aligned}
$$

Therefore, it follows from $(7.20),(7.21)$ and (7.22) that

$$
\begin{aligned}
& \quad\left|\Gamma^{a} Q(u, \nabla u)\right|+\left|\Gamma^{a} \operatorname{rot}(\operatorname{div} u \operatorname{rot} u)\right| \\
& \leq C_{N}\left\{(1+t+|x|)^{-1}\left(z_{\mu, \mu}^{(1)}(t,|x|)^{-1}+z_{\mu, \mu}^{(2)}(t,|x|)^{-1}\right)+\right. \\
& \left.+(1+|x|)^{-1} z_{\mu, \mu}^{(0)}(t,|x|)^{-1}\right\}\left(\varepsilon+\left.[\nabla u]_{[(N+3) / 2], t}|| \nabla u\right|_{N+5, t} ^{2}\right) \\
& \quad \text { for }|a| \leq N \text { and for some } \mu>1 .
\end{aligned}
$$

Since (7.23) gives the estimates of $F_{2}$ in (7.15), we find from (7.6), (7.7), (7.15) and Proposition 6.3 (ii) that

$$
\begin{aligned}
&\left|\Gamma^{a} \operatorname{rot} u\right| \\
& \leq C_{N} u_{2}(t,|x|)^{-1}\left(\varepsilon+[\nabla u]_{[(N+4) / 2], t}|| \nabla u \|_{N+6, t}^{2}\right),
\end{aligned}
$$


which implies

$$
\begin{aligned}
& \left.\left|\Gamma^{a} \operatorname{grad}\right| \operatorname{rot} u\right|^{2} \mid \\
& \leq C_{N}\left\{(1+|x|)^{-1} z_{3 / 2,3 / 2}^{(0)}(t,|x|)+(1+t+|x|)^{-1} z_{2,1}^{(2)}(t,|x|)\right\} \times \\
& \times\left(\varepsilon+[\nabla u]_{[(N+4) / 2], t}|| \nabla u \|_{N+6, t}^{4}\right) \\
& \quad \text { for }|a| \leq N .
\end{aligned}
$$

Since (7.23) and (7.25) give the estimates of $F_{1}$ in (7.14), we also find from (7.6), (7.7), (7.14) and Proposition 6.3 (ii) (iii) that, for $|a| \leq N$,

$$
\begin{aligned}
& \left|\Gamma^{a} \operatorname{div} u\right| \\
\leq & C_{N} w_{1}(t,|x|)^{-1}\left(\varepsilon+[\nabla u]_{[(N+5) / 2], t}\|\nabla u\|_{N+7, t}^{4}\right)
\end{aligned}
$$

Applying $\tilde{\Gamma}^{a}$ to (7.7), we find from (4.1), (5.2) and (7.2) that

$$
\begin{aligned}
& L \tilde{\Gamma}^{a} u_{1}=\tilde{\Gamma}^{a} Q(u, \nabla u) \\
& +2\left(\sigma_{11}-\sigma_{12}\right)\left\{\operatorname{grad} \Gamma^{a}|\operatorname{rot} u|^{2}-2 \tilde{\Gamma}^{a} \operatorname{rot}(\operatorname{div} u \operatorname{rot} u)\right\} \\
& \tilde{\Gamma}^{a} u_{1}(0, x)=\partial_{t} \tilde{\Gamma}^{a} u_{1}(0, x)=0
\end{aligned}
$$

Making use of (6.13) and (6.17),

$$
\begin{aligned}
& \nabla \tilde{\Gamma}^{a} u_{1}(t, x)=I_{1}\left(\nabla \operatorname{grad} \Gamma^{a}|\operatorname{rot} u|^{2}\right)(t, x) \\
& +I\left(-2 \nabla \tilde{\Gamma}^{a} \operatorname{rot}(\operatorname{div} u \operatorname{rot} u)+\nabla \tilde{\Gamma}^{a} Q(u, \nabla u)\right)(t, x),
\end{aligned}
$$

where we normalize $2\left(\sigma_{11}-\sigma_{12}\right)=1$ for simplicity. Since (7.23) and (7.25) give the estimates of the nonlinear terms in (7.27), we find from (7.3), (7.28), Proposition 6.2 (iii) and Proposition 6.3 (ii) (iii) that, for $|a| \leq N$,

$$
\begin{aligned}
& \left|\nabla \Gamma^{a} u(t, x)\right| \\
\leq & C_{N}^{\gamma} w(t,|x|)^{-1}\left(\varepsilon+[\nabla u]_{[(N+5) / 2], t}|| \nabla u \|_{N+7, t}^{4}\right),
\end{aligned}
$$

which implies the estimate (7.1).

\section{Energy estimates and proof of Theorem 3.1}


John [6], [10] has proved that the initial value problem (4.1) has a unique local in time solution $u(t, x)$ of class $C^{\infty}$ and $u(t, x)$ is of compact support in $x$. More precisely, if

$$
f(x)=g(x)=0 \quad \text { for }|x|>R
$$

then

$$
u(t, x)=0 \text { for }|x|>R+c_{1} t
$$

We first prove the following

Lemma 8.1 Let $u$ be the solution of (4.1). Then there exist positive constants $\lambda$ and $C_{N}$ such that

$$
\|\partial u\|_{N, t}^{2} \leq C_{N} \varepsilon^{2}(1+t)^{C_{N}[\nabla u]_{(N+1) / 2], t}}
$$

provided $|\nabla u(s, x)|<\lambda$ for $0 \leq s \leq t, x \in \mathbb{R}^{3}$.

Proof. The nonlinear term $F\left(\nabla u, \nabla^{2} u\right)$ in (4.1) can be written in the form

$$
F^{i}\left(\nabla u, \nabla^{2} u\right)=\sum_{j, \ell, m=1}^{3} D_{i j}^{\ell m}(\nabla u) \partial_{\ell} \partial_{m} u^{j}
$$

where each $D_{i j}^{\ell m}(\nabla u)$ is a first order homogeneous polynomial of $\nabla u$. Proposition 2.1 and Remark 3.3 yield that each matrix $D^{\ell m}(\nabla u)=\left(D_{i j}^{\ell m}(\nabla u)\right)$ is symmertic. Corresponding to a given solution $u(t, x)$ of (4.1) we introduce the linear differential operator matrix

$$
\mathcal{L}=L-\sum_{\ell, m} D^{\ell m}(\nabla u) \partial_{\ell} \partial_{m}
$$

Making use of the symmetric condition on the $C^{\ell m}(\nabla u)$, we obtain an identity valid for vectors $v(t, x)$ (see $[10])$ :

$$
\sum_{\alpha=0}^{3} \partial_{\alpha} Q_{\alpha}(\partial v)=2^{t}\left(\partial_{0} v\right) \mathcal{L} v+q
$$


where $Q$ and $q$ are quadratic forms in the first derivatives of the components $v^{i}$ of the vector $v$ :

$$
\begin{aligned}
Q_{0}= & \left|\partial_{0} v\right|^{2}+c_{2}^{2}|\nabla v|^{2}+\left(c_{1}^{2}-c_{2}^{2}\right)(\operatorname{div} v)^{2} \\
& +\sum_{\ell, m=1}^{3}{ }^{t}\left(\partial_{\ell} v\right) C^{\ell m}(\nabla u) \partial_{m} v, \\
Q_{j}= & -2 c_{2}^{2}{ }^{t}\left(\partial_{0} v\right)\left(\partial_{j} v\right)-2\left(c_{1}^{2}-c_{2}^{2}\right) \operatorname{div} v \partial_{0} v_{j} \\
& -2 \sum_{k=1}^{3}{ }^{t}\left(\partial_{0} v\right) C^{j k}(\nabla u) \partial_{k} v, \\
q= & -2 \sum_{\ell, m=1}^{3}{ }^{t}\left(\partial_{0} v\right)\left(\partial_{\ell} C^{\ell m}(\nabla u)\right) \partial_{m} v .
\end{aligned}
$$

Here ${ }^{t} v$ again denotes the transpose of $v$.

We find easily from (8.5) that there exist positive number $\nu, \mu$ depending on $c_{1}, c_{2}$ and $\lambda$ such that

$$
\nu|\partial v|^{2} \leq Q_{0} \leq \mu|\partial v|^{2} \text { for }|\nabla u|<\lambda \text { and all } v
$$

By integrating the identity (8.4) over $\mathbb{R}^{3}$ we find from (8.8) that

$$
\|\partial v(t)\|_{0}^{2} \leq \frac{\mu}{\nu}\|\partial v(0)\|_{0}^{2}+\frac{1}{\nu} \int_{0}^{t} d s \int_{\mathbb{R}^{3}}\left(2^{t}\left(\partial_{0} v\right) \mathcal{L} v+q\right) d x .
$$

We apply (8.9) to $v=\tilde{\Gamma}^{a} u,|a| \leq N$ and sum over $a$. Since

$$
\mathcal{L}\left(\tilde{\Gamma}^{a} u\right)=\sum_{\ell, m=1}^{3}\left[\tilde{\Gamma}^{a}, C^{\ell m}(\nabla u) \partial_{\ell} \partial_{m}\right] u
$$

it follows from (8.7) that

$$
\begin{aligned}
& \left|\mathcal{L}\left(\tilde{\Gamma}^{a} u\right)\right| \leq C_{N}|\nabla u|_{[(N+1) / 2]}|\partial u|_{N} \\
& \left|q\left(\tilde{\Gamma}^{a} u\right)\right| \leq C|\nabla u|_{1}\left|\partial \tilde{\Gamma}^{a} u\right|^{2}
\end{aligned}
$$

Therefore, we find from (8.9) and them that

$$
\begin{aligned}
\| \partial u(t) & \|_{N}^{2} \leq C_{N}\left(\|\partial u(0)\|_{N}^{2}+\right. \\
& \left.+[\nabla u]_{[(N+2) / 2], t} \int_{0}^{t}(1+s)^{-1}\|\partial u(s)\|_{N}^{2} d s\right)
\end{aligned}
$$


which implies (8.1) using Gronwall inequality.

We next prove the following proposition which guarantee together with Proposition 7.1 the global existence of solutions with small data.

Proposition 8.1. Let $u$ be the solution of (4.1). Then there exists positive constans $\lambda_{N} \leq 1$ and $C_{N}^{\prime \prime}$ such that

$$
\|\partial u\|_{N, t} \leq C_{N}^{\prime \prime} \varepsilon
$$

provided $[\nabla u]_{[(N+6) / 2], t} \leq \lambda_{N}$.

Proof. $\quad$ Applying $\tilde{\Gamma}^{a},|a| \leq N$, to (4.1), we have $L \tilde{\Gamma}^{a} u=\tilde{\Gamma}^{a} F\left(\nabla u, \nabla^{2} u\right)$. Integrating the inner product of $\partial_{t} \tilde{\Gamma}^{a} u$ and this equation, we observe that

$$
E\left(\partial \tilde{\Gamma}^{a} u\right)(t)=E\left(\partial \tilde{\Gamma}^{a} u\right)(0)+\int_{0}^{t} d s \int_{\mathbb{R}^{3}} \tilde{\Gamma}^{a} F \cdot \partial_{t} \tilde{\Gamma}^{a} u d x
$$

where

$$
E(\partial u)(s)=\frac{1}{2} \int_{\mathbb{R}^{3}}\left(\left|\partial_{t} u\right|^{2}+c_{2}^{2}|\nabla u|^{2}+\left(c_{1}^{2}-c_{2}^{2}\right)|\operatorname{div} u|^{2}\right)(s, x) d x
$$

The commutation relation (5.2) and integration by parts yield

$$
\int_{\mathbb{R}^{3}} \tilde{\Gamma}^{a} \operatorname{grad}|\operatorname{rot} u|^{2} \cdot \partial_{t} \tilde{\Gamma}^{a} u d x=-\int_{\mathbb{R}^{3}} \Gamma^{a}|\operatorname{rot} u|^{2} \partial_{t} \Gamma^{a} \operatorname{div} u d x
$$

Then we find from (8.14) and the definition of $F$ that the integral in (8.12) is equal to

$$
\begin{aligned}
& \int_{0}^{t} d s \int_{\mathbb{R}^{3}}\left\{\tilde{\Gamma}^{a}\left(2\left(\sigma_{11}-\sigma_{12}\right) \operatorname{rot}(\operatorname{div} u \operatorname{rot} u)+Q(u, \nabla u)\right) \cdot \partial_{t} \tilde{\Gamma}^{a} u\right. \\
& \left.-4\left(\sigma_{11}-\sigma_{12}\right) \Gamma^{a}|\operatorname{rot} u|^{2} \partial_{t} \Gamma^{a} \operatorname{div} u\right\} d x .
\end{aligned}
$$

Making use of (7.13), (7.17), (7.18) and (7.23), one can verify that the integrands in (8.15) are estimated by

$$
\begin{aligned}
& C_{N}(1+s)^{-1-\kappa}|x|^{-2}\left(\sum_{j=0}^{2}\left(1+\left|c_{j} s-\right| x||\right)^{-1-\kappa} \times\right. \\
& \times\left(\varepsilon^{2}+[\nabla u]_{(N+3) / 2], s}\|\nabla u\|_{N+5, s}^{3}\right)
\end{aligned}
$$


for $\kappa>0$, provided $[\nabla u]_{[(N+3) / 2], t} \leq 1$. Therefore, we find from Lemma 8.1, (8.12), (8.13) and (8.16) that

$$
\|\partial u(t)\|_{N}^{2} \leq C_{N} \varepsilon^{2}\left(1+\int_{0}^{t}(1+s)^{-1-\kappa+C_{N}[\nabla u]_{\lfloor(N+6) / 2], s}} d s\right),
$$

which implies Proposition 8.1 , by taking $\lambda_{N}$ as

$$
\lambda_{N} \leq \min \left(1, \lambda, \kappa / 2 C_{N}\right)
$$

Finally, making use of Proposition 7.1 and Proposition 8.1, we will prove Theorem 4.1. Take $C \geq \max \left(C_{13}^{\prime}, C_{20}^{\prime \prime}\right)$ large enough, so that $[\nabla u]_{13,0} \leq C \varepsilon$. Then there exists $T=T(\varepsilon)>0$ such that the solution $u$ of $(4.1)$ in a slab $[0, T] \times \mathbb{R}^{3}$ satisfies $[\nabla u]_{13, T} \leq$ $2 C \varepsilon$. Set $\varepsilon_{0}=\lambda_{20} / 2 C^{4}$. Suppose that $T^{*}$, maximal of $T$ above, is finite for $\varepsilon(0 \leq \varepsilon \leq$ $\left.\varepsilon_{0}\right)$. Then $[\nabla u]_{13, T}$ tends to $2 C \varepsilon$ as $t \rightarrow T^{*}$. However, Proposition 8.1 yields

$$
\|\partial u\|_{20 . t} \leq C_{20}^{\prime \prime} \varepsilon \leq C \varepsilon \text { for } t<T^{*}
$$

because $[\nabla u]_{13, T} \leq \lambda_{20}$ by the choice of $\varepsilon_{0}$. Thus Proposition 7.1 yields

$$
[\nabla u]_{13, T} \leq C \varepsilon+C^{5} \varepsilon^{4} \leq C \varepsilon\left(1+C^{4} \varepsilon_{0} \leq 3 C \varepsilon / 2\right.
$$

which leads to contradiction.

\section{The null condition}

We consider in general the nonlinear term $F\left(\partial u, \partial^{2} u\right)$ containing time derivatives instead of $F\left(\nabla u, \nabla^{2} u\right)$ in (2.8), where $\partial=\left(\partial_{0}, \nabla\right), \partial_{0}=\partial_{t}$. Thus the quasilinear system considered in this section is expressed in the form

$$
\begin{aligned}
& \sum_{j=1}^{3} \sum_{\alpha, \beta=0}^{3} a_{i j}^{\alpha \beta}(\partial u) \partial_{\alpha} \partial_{\beta} u^{j}=0 \quad(i=1,2,3), \\
& a_{i j}^{\alpha \beta}(\partial u)=a_{i j}^{\beta \alpha}(\partial u) .
\end{aligned}
$$


We assume that

$$
\begin{aligned}
& a_{i i}^{00}(0)=1, a_{i i}^{i i}(0)=-c_{1}^{2}, a_{i i}^{j j}(0)=-c_{2}^{2} \quad(i \neq j) \\
& a_{i j}^{i j}(0)=-\left(c_{1}^{2}-c_{2}^{2}\right) / 2(i \neq j) \text { for } i, j=1,2,3 \\
& \text { and } a_{i j}^{\alpha \beta}(0)=0 \text { otherwise, }
\end{aligned}
$$

so that equation (9.1) reduces to the linear elastic wave equation

$$
L u=\partial_{t}^{2} u-c_{2}^{2} \Delta u-\left(c_{1}^{2}-c_{2}^{2}\right) \operatorname{grad} \operatorname{div} u=0
$$

for infinitesimal $u$.

Set

$$
v={ }^{t}\left(v^{1}, v^{2}, v^{3}\right), \quad v^{i}=\left(\partial u^{i}\right) \quad i=1,2,3 .
$$

Then the vector $v \in \mathbb{R}^{12}$ satisfies a system of first order

$$
\sum_{\alpha=0}^{3} a^{\alpha}(v) \partial_{\alpha} v=0
$$

The $12 \times 12$ matrices $a^{\alpha}(v)$ are partitioned into 9 blocks

$$
a^{\alpha}(v)=\left(b_{i j}^{\alpha} ; i \downarrow j \rightarrow 1,2,3\right)
$$

where

$$
\begin{aligned}
& b_{i j}^{0}=\left(\begin{array}{cccc}
a_{i j}^{00} & & 0 & \\
& \delta_{i j} & & \\
0 & & \delta_{i j} & \\
& & & \delta_{i j}
\end{array}\right), b_{i j}^{1}=\left(\begin{array}{cccc}
2 a_{i j}^{10} & a_{i j}^{11} & a_{i j}^{12} & a_{i j}^{13} \\
-\delta_{i j} & & 0 & \\
0 & 0 & \\
0 & &
\end{array}\right), \\
& b_{i j}^{2}=\left(\begin{array}{cccc}
2 a_{i j}^{20} & a_{i j}^{21} & a_{i j}^{22} & a_{i j}^{23} \\
0 & & & \\
-\delta_{i j} & & 0 & \\
0 & & &
\end{array}\right), b_{i j}^{3}=\left(\begin{array}{cccc}
2 a_{i j}^{30} & a_{i j}^{31} & a_{i j}^{32} & a_{i j}^{33} \\
0 & & & \\
0 & & 0 & \\
-\delta_{i j} & & &
\end{array}\right) \text {. }
\end{aligned}
$$

We next consider the plane wave solution $v(t, x)$ of $(9.4)$

$$
v(t, x)=w(t, s) \quad s=\zeta \cdot x
$$


where $\zeta \cdot x$ stands for the inner product of $\zeta, x \in \mathbb{R}^{3}$. Then $w(t, s)$ satisfies a system in one space dimension

$$
\begin{aligned}
& \partial_{t} w+a(w) \partial_{s} w=0 \\
& a(w)=a^{0}(w)^{-1} \sum_{i=1}^{3} \zeta_{i} a^{i}(w) .
\end{aligned}
$$

We shall investigate, near $w=0$, the eigenvalues $\lambda=\lambda(w)$ of the matrix $a(w)$ and corresponding right eigenvector $\xi=\xi(w)$. Set

$$
\begin{aligned}
& a_{i j}=a_{i j}^{00} \lambda-2 \sum_{k=1}^{3} a_{i j}^{k 0} \zeta_{k}, \\
& a_{i j}^{k}=-\sum_{\ell=1}^{3} a_{i j}^{k \ell} \zeta_{\ell} .
\end{aligned}
$$

Then it follows from (9.5), (9.7) and (9.8) that

$$
a^{0}(w) \lambda-\sum_{i=1}^{3} \zeta_{i} a^{i}(w)=\left(b_{i j}(\lambda)\right)
$$

where

$$
b_{i j}(\lambda)=\left(\begin{array}{cccc}
a_{i j} & a_{i j}^{1} & a_{i j}^{2} & a_{i j}^{3} \\
\zeta_{1} \delta_{i j} & \lambda \delta_{i j} & 0 & 0 \\
\zeta_{2} \delta_{i j} & 0 & \lambda \delta_{i j} & 0 \\
\zeta_{3} \delta_{i j} & 0 & 0 & \lambda \delta_{i j}
\end{array}\right)
$$

By adding to the first, fifth, nineth column of the matrix $(9.9)(1+i)$-th, $(5+i)$-th, $(a+i)$-th, column multiplied by $-\lambda^{-1} \zeta_{i}(i=1,2,3)$, respectively, we find from (9.8), (9.9) and (9.10) that

$$
\operatorname{det}\left(a^{0}(w) \lambda-\sum_{i=1}^{3} \zeta_{i} a^{i}(w)\right)=\lambda^{6} \operatorname{det}\left(p_{i j}(\lambda)\right)
$$

where

$$
\begin{aligned}
p_{i j}(\lambda) & =a_{i j} \lambda-\sum_{k=1}^{3} a_{i j}^{k} \zeta_{k} \\
& =a_{i j}^{00} \lambda^{2}-2 \lambda \sum_{k=1}^{3} a_{i j}^{k 0} \zeta_{k}+\sum_{k, \ell=1}^{3} a_{i j}^{k \ell} \zeta_{k} \zeta_{\ell} .
\end{aligned}
$$


From the assumption (9.2) on $a_{i j}^{\alpha \beta}(0)$ and (9.12), we get, at $w=0$,

$$
\begin{aligned}
& p_{i i}(\lambda)=\lambda^{2}-c_{2}^{2}|\zeta|^{2}-\left(c_{1}^{2}-c_{2}^{2}\right) \zeta_{i}^{2}, \\
& p_{i j}(\lambda)=\left(c_{1}^{2}-c_{2}^{2}\right) \zeta_{i} \zeta_{j} \quad(i \neq j),
\end{aligned}
$$

which implies

$$
\operatorname{det}\left(a^{0}(0) \lambda-\sum_{i=1}^{3} \zeta_{i} a^{i}(0)\right)=\lambda^{6}\left(\lambda^{2}-c_{1}^{2}|\zeta|^{2}\right)\left(\lambda^{2}-c_{2}^{2}|\zeta|^{2}\right)^{2} .
$$

Therefore, we find from (9.11) and (9.14) that there exist eigenvalues $\lambda_{1}^{ \pm}(w), \lambda_{2,1}^{ \pm}(w)$ and $\lambda_{2,2}^{ \pm}(w)$, aside from the trivial multiple eigenvalue $\lambda=0$, such that

$$
\lambda_{1}^{ \pm}(0)= \pm c_{1}|\zeta|, \lambda_{2,1}^{ \pm}(0)=\lambda_{2,2}^{ \pm}(0)= \pm c_{2}|\zeta|
$$

We look for the eigenvectors $\xi=\xi(0)$ corresponding to the eigenvalues $0, \pm c_{i}|\zeta|$. Making use of the assumption (9.2) on $a_{i j}^{\alpha \beta}(0)$, we have from (9.8) and (9.10) that the matrices $b_{i j}(\lambda)$ at $w=0$ are in the forms:

$$
b_{i i}(\lambda)=\left(\begin{array}{cccc}
\lambda & & d_{i}(\zeta) & \\
\zeta_{1} & \lambda & 0 & 0 \\
\zeta_{2} & 0 & \lambda & 0 \\
\zeta_{3} & 0 & 0 & \lambda
\end{array}\right)
$$

with

$$
\begin{aligned}
& d_{1}(\zeta)=\left(c_{1}^{2} \zeta_{1}, c_{2}^{2} \zeta_{2}, c_{2}^{2} \zeta_{3}\right) \\
& d_{2}(\zeta)=\left(c_{2}^{2} \zeta_{1}, c_{1}^{2} \zeta_{2}, c_{2}^{2} \zeta_{3}\right) \\
& d_{3}(\zeta)=\left(c_{2}^{2} \zeta_{1}, c_{2}^{2} \zeta_{2}, c_{1}^{2} \zeta_{3}\right)
\end{aligned}
$$

and the first row $d_{12}(\zeta), d_{13}(\zeta)$ and $d_{23}(\zeta)$ of

$$
b_{12}(\lambda)=b_{21}(\lambda), b_{13}(\lambda)=b_{31}(\lambda) \quad \text { and } \quad b_{23}(\lambda)=b_{32}(\lambda) \text {, }
$$

respectively, is only non-zero and

$$
\begin{aligned}
& d_{12}(\zeta)=\left(c_{1}^{2}-c_{2}^{2}\right)(0,1,1,0) / 2 \\
& d_{13}(\zeta)=\left(c_{1}^{2}-c_{2}^{2}\right)(0,1,0,1) / 2 \\
& d_{23}(\zeta)=\left(c_{1}^{2}-c_{2}^{2}\right)(0,0,1,1) / 2
\end{aligned}
$$


According to (9.3) we arrange the components of $\xi$ as follows,

$$
\xi=\left(\xi^{1} ; \xi^{2} ; \xi^{3}\right), \xi^{i}=\left(\xi_{0}^{i}, \xi_{1}^{i}, \xi_{2}^{i}, \xi_{3}^{i}\right)
$$

Then, from (9.10) and (9.16), one can verify after a bit of calculation that the eigenvectors $\xi_{1}^{ \pm}$and $\xi_{2}^{ \pm}$corresponding to $\pm c_{1}|\zeta|$ and $\pm c_{2}|\zeta|$ are

$$
\xi_{1}^{ \pm}=\left(\mp c_{1}|\zeta| \zeta_{1}, \zeta_{1} \zeta_{i} ; \mp c_{1}|\zeta| \zeta_{2}, \zeta_{2} \zeta_{i} ; \mp c_{1}|\zeta| \zeta_{3}, \zeta_{3} \zeta_{i}\right)
$$

and

$$
\begin{gathered}
\xi_{2}^{ \pm}=\left(\mp c_{2}|\zeta| \xi_{1}, \xi_{1} \zeta_{i} ; \mp c_{2}|\zeta| \xi_{2}, \xi_{2} \zeta_{i} ; \mp c_{2}|\zeta| \xi_{3}, \xi_{3} \zeta_{i}\right) \\
\text { for } \xi \cdot \zeta=0,
\end{gathered}
$$

respectively. The eigenvectors corresponding to $\lambda=0$ are

$$
\begin{aligned}
& (0, \xi ; 0 ; 0),(0 ; 0, \xi ; 0),(0 ; 0 ; 0, \xi) \\
& \quad \text { for } \xi \cdot \zeta=0 .
\end{aligned}
$$

Therefore, we observe from (9.17)-(9.19) that there exist twelve linearly independent eigenvectors at $w=0$. Thus the system (9.7) is strongly hyperbolic near $w=0$.

We also look for the gradients $\nabla_{w} \lambda_{1}^{ \pm}$and $\nabla_{w}\left(\lambda_{21}^{ \pm}+\lambda_{2,2}^{ \pm}\right)$at $w=0$. From (9.13) and (9.15) we have

$$
\begin{aligned}
& p_{i i}\left(\lambda_{1}^{ \pm}(0)\right)=\left(c_{1}^{2}-c_{2}^{2}\right)\left(|\zeta|^{2}-\zeta_{i}^{2}\right), \\
& p_{i j}\left(\lambda_{1}^{ \pm}(0)\right)=-\left(c_{1}^{2}-c_{2}^{2}\right) \zeta_{i} \zeta_{j} \quad(i \neq j)
\end{aligned}
$$

which leads to

$$
\operatorname{cof}\left(p_{i j}\left(\lambda_{1}^{ \pm}(0)\right)=\left(\left(c_{1}^{2}-c_{2}^{2}\right)|\zeta|^{2} \zeta_{i} \zeta_{j}\right)\right.
$$

Then, differentiating $\operatorname{det}\left(p_{i j}\left(\lambda_{1}^{ \pm}(w)\right)\right)=0$ in $w_{\alpha}^{i}$ and evaluating after at $w=0$, we obtain

$$
\sum_{i, j=1}^{3} D\left(\left.p_{i j}\left(\lambda_{1}^{ \pm}(w)\right)\right|_{w=0} \zeta_{i} \zeta_{j}=0\right.
$$


where $D$ denotes one of $\partial / \partial w_{\alpha}^{i}$. From (9.12) and (9.20), we have

$$
\begin{aligned}
& \pm 2 c_{1}|\zeta|^{3}\left(D \lambda_{1}^{ \pm}\right)(0)=-\sum_{i, j} \zeta_{i} \zeta_{j}\left\{c_{1}^{2}|\zeta|^{2}\left(D a_{i j}^{00}\right)(0)\right. \\
& \left.\mp 2 c_{1}|\zeta| \sum_{k}\left(D a_{i j}^{k 0}\right)(0) \zeta_{k}+\sum_{k, \ell}\left(D a_{i j}^{k \ell}\right)(0) \zeta_{k} \zeta_{\ell}\right\}
\end{aligned}
$$

From (9.21), (9.13) and (9.15),

$$
\begin{aligned}
& p_{i j}\left(\lambda_{2, k}^{ \pm}(0)\right)=-\left(c_{1}^{2}-c_{2}^{2}\right) \zeta_{i} \zeta_{j} \\
& \operatorname{cof}\left(p_{i j}\left(\lambda_{2, k}^{ \pm}(0)\right)=(0)\right. \\
& \partial_{\lambda} p_{i i}\left(\lambda_{2, k}^{ \pm}(0)\right)= \pm c_{2}|\zeta| \\
& \partial_{\lambda} p_{i j}\left(\lambda_{2, k}^{ \pm}(0)\right)=0 \quad(i \neq j)
\end{aligned}
$$

for $k=1,2$. Assume that $D\left(\lambda_{\mathbf{2}, \mathbf{1}}^{ \pm}(w)+\lambda_{\mathbf{2}, 2}^{ \pm}(w)\right)$ exists at $w=0$. Then, differentiating the identity

$$
\left.\partial_{\lambda} \operatorname{det}\left(p_{i j}(\lambda)\right)\right|_{\lambda=\lambda_{2,1}^{ \pm}(w)}+\left.\partial_{\lambda} \operatorname{det}\left(p_{i j}(\lambda)\right)\right|_{\lambda=\lambda_{2,2}^{ \pm}(w)}=0
$$

and evaluating at $w=0$, one can verify after a bit of calculation that

$$
\begin{aligned}
& \left.\sum_{i} D\left(p_{i i}\left(\lambda_{2,1}^{ \pm}\right)+p_{i i}\left(\lambda_{2,2}^{ \pm}\right)\right)\right|_{w=0}\left(|\zeta|^{2}-\zeta_{i}^{2}\right)+ \\
& +\left.\sum_{i \neq j} D\left(p_{i j}\left(\lambda_{2,1}^{ \pm}\right)+p_{i j}\left(\lambda_{2,2}^{ \pm}\right)\right)\right|_{w=0} \zeta_{i} \zeta_{j}=0 .
\end{aligned}
$$

From (9.12) and (9.22), we have

$$
\begin{aligned}
& \pm\left. 2 c_{2}|\zeta|^{2} D\left(\lambda_{2,1}^{ \pm}(w)+\lambda_{2,2}^{ \pm}(w)\right)\right|_{w=0} \\
& =-\sum_{i}\left(|\zeta|^{2}-\zeta_{i}^{2}\right)\left\{c_{2}|\zeta|^{2}\left(D a_{i i}^{00}\right)(0) \mp c_{2}|\zeta| \sum_{k}\left(D a_{i i}^{k 0}\right)(0) \zeta_{k}\right. \\
& \left.+\sum_{k, \ell}\left(D a_{i i}^{k \ell}\right)(0) \zeta_{k} \zeta_{\ell}\right\}-\sum_{i \neq j} \zeta_{i} \zeta_{j}\left\{c_{2}|\zeta|^{2}\left(D a_{i j}^{00}\right)(0)\right. \\
& \left.\mp c_{2}|\zeta| \sum_{k}\left(D a_{i j}^{k 0}\right)(0) \zeta_{k}+\sum_{k, \ell}\left(D a_{i j}^{k \ell}\right)(0) \zeta_{k} \zeta_{\ell}\right\}
\end{aligned}
$$

Therefore, we conclude that the derivative $D\left(\lambda_{2,1}^{ \pm}(w)+\lambda_{2,2}^{ \pm}(w)\right)$ exists at $w=0$ and is determined by (9.23). 
Now we made ready to state the genuien nonlinearity.

Definition 9.1. We say that the system (9.7) for fixed $\zeta \neq 0$ is not genuienly nonlinear if and only if

$$
\left.\xi_{1}^{ \pm} \cdot \nabla_{w} \lambda_{1}^{ \pm}\right|_{w=0}=0 \quad \text { and }\left.\xi_{2}^{ \pm} \cdot \nabla_{w}\left(\lambda_{2,1}^{ \pm}+\lambda_{2,2}^{ \pm}\right)\right|_{w=0}=0
$$

where $\xi_{1}^{ \pm}$and $\xi_{2}^{ \pm}$are defined in (9.17) and (9.18), respectively.

We will prove the following

Proposition 9.1. The quasilinear system (9.7) is not genuienly nonlinear for any $\zeta \neq 0$ if and only if

$(\mathrm{N})_{1}$

$$
\begin{aligned}
& \left.\sum_{i, j, k=1}^{3} \sum_{\alpha, \beta, \gamma=0}^{3} \frac{\partial a_{i j}^{\alpha \beta}(\partial u)}{\partial\left(\partial_{\gamma} u^{k}\right)}\right|_{\partial u=0} X_{i} X_{j} X_{k} X_{\alpha} X_{\beta} X_{\gamma}=0 \\
& \text { for }\left(X_{0}, X_{1}, X_{2}, X_{3}\right) \text { satisfying } X_{0}^{2}-c_{1}|X|^{2}=0
\end{aligned}
$$

and

$(\mathrm{N})_{2}$

$$
\left.\sum_{i, k=1}^{3} \sum_{\alpha, \beta, \gamma=0}^{3} \frac{\partial a_{i i}^{\alpha \beta}(\partial u)}{\partial\left(\partial_{\gamma} u^{k}\right)}\right|_{\partial u=0}\left(|X|^{2}-X_{i}^{2}\right) \xi_{k} X_{\alpha} X_{\beta} X_{\gamma}
$$

$$
-\left.\sum_{\substack{i, j, k=1 \\ i \neq j}}^{3} \sum_{\alpha, \beta, \gamma=0}^{3} \frac{\partial a_{i j}^{\alpha \beta}(\partial u)}{\partial\left(\partial_{\gamma} u^{k}\right)}\right|_{\partial u=0} X_{i} X_{j} \xi_{k} X_{\alpha} X_{\beta} X_{\gamma}=0
$$

for $\xi,\left(X_{0}, X_{1}, X_{2}, X_{3}\right)$ satisfying $X_{0}^{2}-c_{2}|X|^{2}=0$ and $\xi \cdot X=0$.

We call the condition, $(\mathrm{N})_{1}$ and $(\mathrm{N})_{2}$, the null condition for the quasilinear system (9.1).

Proof of Proposition 9.1. Set

$$
X_{0}=\mp c_{1}|\zeta|, X_{i}=\zeta_{i} \quad(i=1,2,3) .
$$

Then, from (9.17) and (9.21), we rewrite $\xi_{1}^{ \pm}$and $\left(D \lambda_{1}^{ \pm}\right)(0)$ in the form

$$
\begin{aligned}
& \xi_{1}^{ \pm}=\left(X_{1}\left(X_{0}, X_{i}\right) ; X_{2}\left(X_{0}, X_{i}\right) ; X_{3}\left(X_{0}, X_{i}\right)\right) \\
& \pm 2 c_{1}|\zeta|^{3}\left(D \lambda_{1}^{ \pm}(0)\right)=-\sum_{i, j=1}^{3} X_{i} X_{j} \sum_{\alpha, \beta=0}^{3}\left(D a_{i j}^{\alpha \beta}\right)(0) X_{\alpha} X_{\beta} .
\end{aligned}
$$


Therefore, from the first condition of (9.24), we obtain the condition $(\mathrm{N})_{1}$. Similarly, we set

$$
X_{0}=\mp c_{2}|\zeta|, X_{i}=\zeta_{i} \quad(i=1,2,3) .
$$

Then, from (9.18) and (9.23), we rewrite $\xi_{2}^{ \pm}$and $\left.D\left(\lambda_{2,1}^{ \pm}(w)+\lambda_{2,2}^{ \pm}(w)\right)\right|_{w=0}$ in the form

$$
\begin{aligned}
& \quad \xi_{2}^{ \pm}=\left(\xi_{1}\left(X_{0}, X_{i}\right) ; \xi_{2}\left(X_{0}, X_{i}\right) ; \xi_{3}\left(X_{0}, X_{i}\right)\right) \\
& \quad \text { for } \xi_{1} X_{1}+\xi_{2} X_{2}+\xi_{3} X_{3}=0 \\
& \pm\left. c_{2}|\zeta|^{2} D\left(\lambda_{2,1}^{ \pm}(w)+\lambda_{2,2}^{ \pm}(w)\right)\right|_{w=0} \\
& =-\sum_{i}\left(|X|^{2}-X_{i}^{2}\right) \sum_{\alpha, \beta=0}^{3}\left(D a_{i i}^{\alpha \beta}\right)(0) X_{\alpha} X_{\beta}-\sum_{i \neq j} X_{i} X_{j} \sum_{\alpha, \beta=0}^{3}\left(D a_{i j}^{\alpha \beta}\right)(0) X_{\alpha} X_{\beta} .
\end{aligned}
$$

Therefore, from the second condition of $(9.24)$, we obtain the condition $(\mathrm{N})_{2}$.

\section{Proof of Proposition 6.2.}

In order to Proposition 6.2, we shall discuss the integral in the form

$$
J_{a}(g(y) ; x, \tau)=\frac{\tau}{4 \pi} \int_{|\omega|=1} \omega^{a} g(x+\tau \omega) d S_{\omega} .
$$

Then, the integral $I(F)(t, x)$ defined in Section 6 can be expressed by a linear combination of the followings:

$$
\begin{aligned}
& \int_{0}^{t} J_{a}\left(F(s, y) ; x, c_{i}(t-s)\right) d s \\
& \int_{0}^{t}(t-s) d s \int_{c_{2}(t-s)}^{c_{1}(t-s)} \tau^{-2} J_{a}(F(s, y) ; x, \tau) d \tau \\
& \quad(i=1,2 \text { and }|a|=0,2) .
\end{aligned}
$$

Let $A$ be an orthogonal matrix with $x=A(0,0, r),|x|=r$. By change of variables $\omega=A \zeta$ and using the standard spherical coordinates $\theta, \varphi$ on the sphere of radius $\tau$ and center $(0,0, r)$,

$$
\begin{aligned}
& J_{a}(g(y) ; x, \tau)=\frac{\tau}{4 \pi} \int_{0}^{\pi} \sin \theta d \theta \int_{0}^{2 \pi}(A \zeta)^{a} \times \\
& \times(g \circ A)(\tau \sin \theta \cos \varphi, \tau \sin \theta \sin \varphi, r+\tau \cos \varphi) d \varphi
\end{aligned}
$$


Moreover, following Appendix of [8], introducing an angle $\psi$ between two vectors $x$ and $x+\tau \omega$ so that

$$
x \cdot(x+\tau \omega)=r \lambda \cos \psi, \lambda=|x+\tau \omega|
$$

we then find that

$$
\begin{aligned}
& \tau \sin \theta=\lambda \sin \psi, r+\tau \cos \theta=\lambda \cos \psi, \\
& \lambda^{2}=r^{2}+\tau^{2}+2 r \tau \cos \theta, \\
& \tau^{2}=\lambda^{2}+r^{2}-2 r \lambda \cos \psi .
\end{aligned}
$$

Set

$$
\begin{aligned}
\Theta & =\Theta(\lambda, \varphi, \tau) \\
& =A(\sin \psi \cos \varphi, \sin \psi \sin \varphi, \cos \psi)
\end{aligned}
$$

and

$$
\Xi=\Xi(\lambda, \varphi, \tau)=\tau^{-1}(\lambda \Theta-x)
$$

Introducing a new independent variable $\lambda$ instead of $\theta$ in the integral (10.4), one can verify from (10.4)-(10.7) that

$$
J_{a}(g(y) ; x, \tau)=\frac{1}{4 \pi r} \int_{|r-\tau|}^{r+\tau} \lambda d \lambda \int_{0}^{2 \pi} \Xi^{a} g(\lambda \Theta) d \varphi .
$$

We begin to show Proposition 6.2 (i). Proposition 6.2 (i) for (10.2) has been proved in Proposition 3.1 of [16], because $\omega$ is a unit vector. Making use of (6.14), (10.3) and (10.11), we observve that Proposition 6.2 (i) follows from

$$
I_{0} \leq C(1+t+r)^{-1}(\log (2+t))^{2}
$$

where

$$
\begin{gathered}
I_{0}=\frac{1}{4 \pi r} \int_{0}^{t}(t-s) d s \int_{c_{2}(t-s)}^{c_{1}(t-s)} \tau^{-2} d \tau \int_{|r-\tau|}^{r+\tau} z_{1,1}^{(j)}(s, \lambda) d \lambda \\
(j=0,1,2) .
\end{gathered}
$$


Here and hereafter, we denote by $C$ a various positive constant depending on $c_{1}, c_{2}$ and $\mu$.

We will prove (10.12) by separating five cases.

Case 1. $r \leq 1$.

Since $r+\tau-|r-\tau| \leq 2 r \leq 2$, we have

$$
\begin{aligned}
I_{0} & \leq \frac{9}{2 \pi} \int_{0}^{t}(t-s) d s \int_{c_{2}(t-s)}^{c_{1}(t-s)} \tau^{-2}\left(1+\left|c_{j} s-r-\tau\right|\right)^{-1}\left(1+c_{1} s+r+\tau\right)^{-1} d \tau \\
& \leq \frac{9}{2 \pi}\left(1+c_{2} t+r\right)^{-1} \int_{0}^{t}(t-s) d s \int_{c_{2}(t-s)}^{c_{1}(t-s)} \tau^{-2}\left(1+\left|c_{j} s-r-\tau\right|\right)^{-1} d \tau
\end{aligned}
$$

Integration by parts in $\tau$ yields

$$
I_{0} \leq C(1+t+r)^{-1} \log (2+t)
$$

Case 2. $r \geq 1, r \geq 2 c_{1} t$.

In this case, $|r-\tau|=r-\tau$. We then have

$$
\begin{aligned}
& c_{1} s+\lambda \geq c_{1} s+r-\tau \geq c_{1} s+r-c_{1}(t-s) \geq r-c_{1} t \geq r / 2, \\
& \left|c_{j} s-\lambda\right| \geq \lambda-c_{j} s \geq r-\tau-c_{j} s \geq r-c_{1}(t-s)-c_{j} s \geq r / 2 .
\end{aligned}
$$

Hence,

$$
I_{0} \leq \frac{2 t}{c_{2} \pi(1+r)^{2}} \leq C(1+t+r)^{-1}
$$

Case 3. $r \geq 1, c_{1} t \leq r \leq 2 c_{1} t$ or $r \geq 1, c_{2} t<r<c_{1} t, c_{1}(t-s)<r$.

Extending the domain of $(\tau, \lambda)$-integration to $\left[c_{2}(t-s), c_{1}(t-s)\right] \times\left[r-c_{a}(t-s), r+\right.$ $\left.c_{1}(t-s)\right]$

$$
\begin{aligned}
I_{0} & \leq \frac{1}{4 \pi c_{2} r} \int_{\left(c_{1} t-r\right)_{+} / c_{1}}^{t} d s \int_{r-c_{1}(t-s)}^{r+c_{1}(t-s)}\left(1+\left|c_{j} s-\lambda\right|\right)^{-1}\left(1+c_{1} s+\lambda\right)^{-1} d \lambda \\
& \leq \frac{1}{2 \pi c_{2}}(1+r)^{-1} \int_{0}^{t}\left(1+c_{1} s\right)^{-1} d s \int_{r-c_{1}(t-s)}^{r+c_{1}(t-s)}\left(1+\left|c_{j} s-\lambda\right|\right)^{-1} d \lambda \\
& \leq C(1+t+r)^{-1}(\log (2+t))^{2} .
\end{aligned}
$$


Here $d_{+}=\max (0, d)$.

Case 4. $\quad r \geq 1, c_{2} t \leq r \leq c_{1} t, c_{1}(t-s) \geq r$ or $r \geq 1, r \leq c_{2} t, c_{2}(t-s) \leq r$.

Extending the domain of $(\tau, \lambda)$-integration to $\left[c_{2}(t-s), c_{1}(t-s)\right] \times\left[0, r+c_{1}(t-s)\right]$, $I_{0} \leq \frac{1}{2 \pi c_{2}(1+r)} \int_{\left(c_{2} t-r\right)_{+} / c_{2}}^{\left(c_{1} t-r\right) / c_{1}} d s \int_{0}^{r+c_{1}(t-s)}\left(1+\left|c_{j} s-\lambda\right|\right)^{-1}\left(1+c_{1} s+\lambda\right)^{-1} d \lambda$.

If $c_{2} t / 2 \leq r \leq c_{1} t$, we get in a similar way as Case 3

$$
I_{0} \leq C(1+t+r)^{-1}(\log (2+t))^{2} .
$$

If $r \leq c_{2} t / 2$, then

$$
1+c_{1} s+\lambda \geq 1+c_{1}\left(c_{2} t-r\right) c_{2}^{-1} \geq C(1+t+r)
$$

Hence we obtain

$$
\begin{aligned}
I_{0} & \leq C(1+t+r)^{-1}(1+r)^{-1} \int_{\left(c_{2} t-r\right) / c_{2}}^{\left(c_{1} t-r\right) / c_{1}} d s \int_{0}^{r+c_{1}(t-s)}\left(1+\left|c_{j} s-\lambda\right|\right)^{-1} d \lambda \\
& \leq C(1+t+r)^{-1} \log (2+t) .
\end{aligned}
$$

Case 5. $r \geq 1, r \leq c_{2} t, r<c_{2}(t-s)$.

If $c_{2} t / 2 \leq r \leq c_{2} t$, then, extending the domain of $(\tau, \lambda)$-integration to $\left[c_{2}(t-\right.$ $\left.s), c_{1}(t-s)\right] \times\left[c_{2}(t-s)-r, r+c_{1}(t-s)\right]$, we get in a similar way as Case 3

$$
\begin{aligned}
I_{0} & \leq \frac{1}{2 \pi c_{2}(1+r)} \int_{0}^{\left(c_{2} t-r\right) / c_{2}} d s \int_{c_{2}(t-s)-r}^{r+c_{1}(t-s)}\left(1+\left|c_{j} s-\lambda\right|\right)^{-1}\left(1+c_{1} s+\lambda\right)^{-1} d \lambda \\
& \leq C(1+t+r)^{-1}(\log (2+t))^{2}
\end{aligned}
$$

If $r \leq c_{2} t / 2$, then, extending the domain of $(\tau, \lambda)$ - integration to $\left[c_{2}(t-s), c_{1}(t-s)\right] \times$ $\left[c_{2}(t-s)-r, c_{2}(t-s)+r\right]$ and $\lambda-r \leq \tau \leq \lambda+r, r+c_{2}(t-s) \leq \lambda \leq r+c_{1}(t-s)$,

$$
I_{0} \leq \frac{1}{4 \pi c_{2} r} \int_{0}^{\left(c_{2} t-r\right) / c_{2}} d s \int_{c_{2}(t-s)-r}^{c_{2}(t-s)+r} z_{1,1}^{(j)}(s, \lambda)^{-1} d \lambda
$$

$$
+\frac{1}{\pi r} \int_{c_{2}(t-s)+r}^{\left(c_{2} t-r\right) / c_{2}}(t-s) d s \int_{1,1}^{c_{1}(t-s)+r}(s, \lambda)^{-1} d \lambda \int_{\lambda-r}^{\lambda+r}(1+\tau)^{-2} d \tau
$$


Here we have used that $\tau \geq c_{2}(t-s) \geq r \geq 1$. Since $1+c_{2} s+\lambda \geq 1+c_{2} t+r$ and

$$
\int_{\lambda-r}^{\lambda+r}(1+\tau)^{-2} d \tau \leq 2 r\left(1+c_{2}(t-s)\right)^{-2}
$$

we observe that the second integral of (10.13) is estimated by

$$
\begin{aligned}
& C(1+t+r)^{-1} \int_{0}^{t}(t-s+1)^{-1} d s \int_{c_{2}(t-s)+r}^{c_{1}(t-s)+r}\left(1+\left|c_{j} s-\lambda\right|\right)^{-1} d \lambda \\
\leq & C(1+t+r)^{-1}(\log (2+t))^{2}
\end{aligned}
$$

In order to estimate the first integral of $(10.13)$, we introduce the new variables

$$
\alpha=c_{i} s+\lambda, \beta=-c s+\lambda \quad(i=1,2)
$$

where $c$ stands for one of $c_{j}(j=0,1,2)$. We can verify that, for any function $\varphi(s, \lambda)$,

$$
\int_{0}^{t} d s \int_{\left|r-c_{i}(t-s)\right|}^{r+c_{i}(t-s)} \varphi(s, \lambda) d \lambda=\left(c_{i}+c\right)^{-1} \int_{\left|c_{i} t-r\right|}^{c_{i} t+r} d \alpha \int_{\alpha_{0}}^{\alpha} \varphi(s, \lambda) d \beta
$$

where

$$
\alpha_{0}=\left\{\left(c_{i}-c\right) \alpha+\left(c_{i}+c\right)\left(c_{i} t-r\right)\right\} / 2 c_{i}
$$

Note that

$$
-c \alpha / c_{i} \leq \alpha_{0} \leq \alpha \text { for }\left|c_{i} t-r\right|<\alpha<c_{i} t+r
$$

Since $z_{1,1}^{(j)}(s, \lambda)$ is equivalent to $\left(1+\left|c_{j} s-\lambda\right|\right)\left(1+c_{2} s+\lambda\right)$, we find from (10.15) and (10.17) that the first integral of (10.13) is estiamted by

$$
\begin{aligned}
& C r^{-1} \int_{c_{2} t-r}^{c_{2} t+r}(1+\alpha)^{-1} d \alpha \int_{\alpha_{0}}^{\alpha}(1+|\beta|)^{-1} d \beta \\
\leq & C\left(1+c_{2} t-r\right)^{-1} \log (2+t) \\
\leq & C(1+t+r)^{-1} \log (2+t) .
\end{aligned}
$$


Therefore, we have proved the assertion (10.12).

Next we will prove Proposition 6.2 (ii) (iii) for

$$
\begin{aligned}
& \int_{0}^{t} J_{a}\left((\partial F)(s, y) ; x, c_{i}(t-s)\right) \\
= & \frac{1}{4 \pi r} \int_{0}^{t} d s \int_{\left|r-c_{i}(t-s)\right|}^{r+c_{i}(t-s)} \lambda d \lambda \int_{0}^{2 \pi}\left(\Xi^{(i)}\right)^{a}(\partial F)\left(s, \lambda \Theta^{(i)}\right) d \varphi .
\end{aligned}
$$

Here we have used the formula (10.11) and

$$
\begin{aligned}
& \Theta^{(i)}(\lambda, \varphi, s)=\Theta\left(\lambda, \varphi, c_{i}(t-s)\right), \\
& \Xi^{(i)}(\lambda, \varphi, s)=\Xi\left(\lambda, \varphi, c_{i}(t-s)\right) .
\end{aligned}
$$

To this end we make use of the following formula

$$
\begin{aligned}
(\nabla F)(s, \lambda \Theta)= & \Theta \partial_{\lambda}\{F(x, \lambda \Theta)\}+\Theta\left\{\partial_{\lambda} \Theta \cdot(\Theta \wedge(\Omega F)(s, \lambda \Theta))\right\} \\
& -\lambda^{-1} \Theta \wedge(\Omega F)(s, \lambda \Theta) \\
\left(\partial_{t} F\right)\left(s, \lambda \Theta^{(i)}\right)= & \partial_{s}\left(F\left(s, \lambda \Theta^{(i)}\right)-\partial_{s} \Theta^{(i)} \cdot\left(\Theta^{(i)} \wedge \Omega F\right)\left(s, \lambda \Theta^{(i)}\right) .\right.
\end{aligned}
$$

In fact, by (5.1)

$$
(\nabla F)(s, \lambda \Theta)=\Theta\left(\partial_{r} F\right)(s, \lambda \Theta)-\lambda^{-1} \Theta \wedge(\Omega F)(s, \lambda \Theta) .
$$

Since $\partial_{\lambda} \Theta \cdot \Theta=0$, we have

$$
\begin{aligned}
& \left(\partial_{r} F\right)(s, \lambda \Theta) \\
& =\partial_{\lambda}\{F(s, \lambda \Theta)\}-\lambda \partial_{\lambda} \Theta \cdot(\nabla F)(s, \lambda \Theta) \\
& =\partial_{\lambda}\{F(s, \lambda \Theta)\}-\lambda \partial_{\lambda} \Theta \cdot\left\{\Theta\left(\partial_{r} F\right)(s, \lambda \Theta)-\lambda^{-1} \Theta \wedge(\Omega F)(s, \lambda \Theta)\right\} \\
& =\partial_{\lambda}\{F(s, \lambda \Theta)\}+\partial_{\lambda} \Theta \cdot(\Theta \wedge(\Omega F)(s, \lambda \Theta)) .
\end{aligned}
$$

In a similar manner, we obtain the second equality of (10.19).

Let $D^{(i)}$ be the domain of $(s, \lambda)$-integration in (10.18). We split the domain $D^{(i)}$ into $D_{1}^{(i)}$ and $D_{2}^{(i)}$, where

$$
\begin{aligned}
& D_{1}^{(i)}=\left\{(s, \lambda) \in D^{(i)} ; \lambda_{1}^{(i)} \leq \lambda \leq \lambda_{1}^{(i)}+\delta \quad \text { or } \lambda_{2}^{(i)}-\delta \leq \lambda \leq \lambda_{2}^{(i)}\right\}, \\
& D_{2}^{(i)}=D^{(i)} \backslash D_{1}^{(i)}, \quad \delta=\min (1, r), \\
& \lambda_{1}^{(i)}=\left|r-c_{i}(t-s)\right|, \lambda_{2}^{(i)}=r+c_{i}(t-s) .
\end{aligned}
$$


Note that $D_{2}^{(i)}=\emptyset$ if $r<1$. Making use of (10.18) and (10.19) with $\tau=c_{i}(t-s)$, we obtain

$$
\begin{aligned}
& 4 \pi r \int_{0}^{t} J_{a}\left((\nabla F)(s, y) ; x, c_{i}(t-s)\right) d s \\
= & \int_{D_{1}^{(i)}} \lambda d \lambda d s \int_{0}^{2 \pi}\left(\Xi^{(i)}\right)^{a}(\nabla F)\left(s, \lambda \Theta^{(i)}\right) d \varphi \\
& +\int_{\partial D_{2}^{(i)}} n_{\lambda} d \sigma \int_{0}^{2 \pi} \lambda \Theta^{(i)}\left(\Xi^{(i)}\right)^{a} F\left(s, \lambda \Theta^{(i)} d \varphi\right.
\end{aligned}
$$

$$
\begin{aligned}
& -\int_{D_{2}^{(i)}} d \lambda d s \int_{0}^{2 \pi} \partial_{\lambda}\left(\lambda \Theta^{(i)}\left(\Xi^{(i)}\right)^{a}\right) F\left(s, \lambda \Theta^{(i)}\right) d \varphi \\
& +\int_{D_{2}^{(i)}} \lambda d \lambda d s \int_{0}^{2 \pi}\left(\Xi^{(i)}\right)^{a}\left[\Theta^{(i)}\left\{\partial_{\lambda} \Theta^{(i)} \cdot\left(\Theta^{(i)} \wedge \Omega F\right)\left(s, \lambda \Theta^{(i)}\right)\right\}\right. \\
& \left.\quad-\lambda^{-1} \Theta^{(i)} \wedge(\Omega F)\left(s, \lambda \Theta^{(i)}\right)\right] d \varphi \\
& 4 \pi r \int_{0}^{t} J_{a}\left(\partial_{t} F\right)\left(s, y ; x, c_{i}(t-s)\right) d s \\
& =\int_{D_{1}^{(i)}} \lambda d \lambda d s \int_{0}^{2 \pi}\left(\Xi^{(i)}\right)^{a}\left(\partial_{t} F\right)\left(s, \lambda \Theta^{(i)}\right) d \varphi \\
& +\int_{\partial D_{2}^{(i)}} n_{s} d \sigma \int_{0}^{2 \pi} \lambda\left(\Xi^{(i)}\right)^{a} F\left(s, \lambda \Theta^{(i)}\right) d \varphi \\
& \quad-\int_{D_{2}^{(i)}} d \lambda d s \int_{0}^{2 \pi} \partial_{s}\left(\lambda\left(\Xi^{(i)}\right)^{a}\right) F\left(s, \lambda \Theta^{(i)}\right) d \varphi \\
& +\int_{D_{2}^{(i)}} \lambda d \lambda d s \int_{0}^{2 \pi}\left(\Xi^{(i)}\right)^{a} \partial_{s} \Theta^{(i)} \cdot\left(\Theta^{(i)} \wedge \Omega F\right)\left(s, \lambda \Theta^{(i)}\right) d \varphi
\end{aligned}
$$

Here, $\left(n_{\lambda}, n_{s}\right)$ is the unit outer normal to $\partial D_{2}^{(i)}$ and $d \sigma$ is the line element on $\partial D_{2}^{(i)}$.

For $|a|=0$, it has proved in Proposition 3.1 of [16] that Proposition 6.2 (ii), (iii) is valid with $\log (2+t)$ instead of $(\log (2+t))^{2}$. Therefore, since $\Xi^{(i)}$ is a unit vector, it is enough to prove that

$$
\begin{aligned}
& \frac{1}{4 \pi r} \int_{D_{2}^{(i)}} d \lambda d s \int_{0}^{2 \pi} \lambda\left|\left(\partial_{\lambda}+\partial_{s}\right)\left(\left(\Xi^{(i)}\right)^{a}\right) F\left(s, \lambda \Theta^{(i)}\right)\right| d \varphi \\
& \leq C w_{i}(t, x)^{-1}\left(\Phi_{\mu}(t)\right)^{2} M_{\mu, \mu, 0}^{(j)}(F)
\end{aligned}
$$


where

$$
\Phi_{\mu}(t)= \begin{cases}\log (2+t) & \mu=1 \\ 1 & \mu>1 .\end{cases}
$$

for $|a|=2$ and $j=0,1,2$. By the definitions (10.10) and (10.18) of $\Xi$ and $\Xi^{(i)}$,

$$
\begin{aligned}
& \left|\lambda\left(\partial_{\lambda}+\partial_{s}\right)\left(\left(\Xi^{(i)}\right)^{a}\right)\right| \\
& \left.\leq \frac{\lambda}{c_{i}(t-s)}\left(3+\lambda \mid \partial_{\lambda} \Theta^{(i)}\right)|+\lambda| \partial_{s} \Theta^{(i)} \mid\right)
\end{aligned}
$$

Hence, if $\lambda \leq c_{i}(t-s)$, then this term has treated in the case where $|a|=0$. Therefore, we assume hereafter that $\lambda \geq c_{i}(t-s)$.

To estimate (10.23) we make use of the following inequality

$$
\begin{aligned}
& \left|\partial_{\lambda} \Theta\right| \leq 2 \tau h(\lambda, \tau)^{-1 / 2} \text { for }|r-\tau| \leq \lambda \leq|r+\tau| \\
& \left|\partial_{\tau} \Theta\right|=2 \tau h(\lambda, \tau)^{-1 / 2}
\end{aligned}
$$

where

$$
h(\lambda, \tau)=\left(\lambda^{2}-(r-\tau)^{2}\right)\left((r+\tau)^{2}-\lambda^{2}\right)
$$

In fact, differentiating (10.8) and (10.9) in $\lambda$, we have

$$
\partial_{\lambda} \psi=(r \cos \psi-\lambda) / r \lambda \sin \psi, \quad\left|\partial_{\lambda} \Theta\right|=\left|\partial_{\lambda} \psi\right|
$$

Since $r \cos \psi-\lambda=\left(r^{2}-\lambda^{2}-\tau^{2}\right) / 2 \lambda$ and $\sin \psi=h(\lambda, \tau)^{1 / 2} / 2 r \lambda$, we have

$$
\left|\partial_{\lambda} \Theta\right|=\left|\lambda^{2}+\tau^{2}-r^{2}\right| / \lambda h(\lambda, \tau)^{1 / 2}
$$

Thus, (10.24) follows from the fact that

$$
\left|\lambda^{2}+\tau^{2}-r^{2}\right| \leq 2 \lambda \tau \text { for }|r-\tau| \leq \lambda \leq r+\tau \text {. }
$$

In a similar manner, we obtain easily the second equality of (10.24). Set $\tau=c_{i}(t-s)$ in (10.24). Then we have

$$
\frac{\lambda}{c_{i}(t-s)}\left|\partial_{\lambda} \Theta^{(i)}\right| \leq 2\left\{\left(\lambda-\lambda_{1}^{(i)}\right)\left(\lambda_{2}^{(i)}-\lambda\right)\right\}^{-1 / 2}
$$


we can assume that $r, t-s, \lambda-\lambda_{1}^{(i)}, \lambda_{2}^{(i)}-\lambda \geq 1$ in the domain $D_{2}^{(i)}$. Thus, making use of $(6.14),(10.22),(10.25)$, we observe that the estimate (10.22) follows from

$$
\begin{aligned}
& \int_{\bar{D}_{2}^{(i)}}(1+t-s)^{-1} z_{\mu, \mu}^{(j)}(s, \lambda)^{-1} d \lambda d s \\
& +\int_{\bar{D}_{2}^{(i)}}\left\{\left(1+\lambda-\lambda_{1}^{(i)}\right)\left(1+\lambda_{2}^{(i)}-\lambda\right)^{-1 / 2} z_{\mu, \mu}^{(j)}(s, \lambda)^{-1} d \lambda d s\right. \\
& \leq C\left(1+\left|c_{i} t-r\right|\right)^{-1}\left(\Phi_{\mu}(t)\right)^{2}
\end{aligned}
$$

where $\bar{D}_{2}^{(i)}=D_{2}^{(i)} \cap\left\{(s, \lambda) ; \lambda \geq c_{i}(t-s)\right\}$.

We first treat the first integral of (10.26). If $r \geq 2 c_{1} t$, we have

$$
c_{i} s+\lambda,\left|c_{j} s-\lambda\right| \geq r / 2 \quad(j=0,1,2)
$$

which imply $z_{\mu, \mu}^{(j)}(s, \lambda) \geq C(1+r)^{2 \mu}$. Since, $\lambda_{2}^{(i)}-\lambda_{1}^{(i)} \leq 2 r$, the first integral of $(10.26)$ is estimated by

$$
C(1+r)^{-2 \mu} t \leq C(1+t+r)^{-1} \text {. }
$$

In the case where $r \leq 2 c_{1} t$. We make use of new variables $(\alpha, \beta)$ defined in (10.14). It follows from (10.14) and (10.16) that

$$
\begin{aligned}
t-s & =\left\{\left(c_{i}+c\right) t-\alpha+\beta\right\}\left(c_{i}+c\right)^{-1} \\
& \geq\left\{\left(c_{i}+c\right) t-\alpha+\alpha_{0}\right\}\left(c_{i}+c\right)^{-1} \\
& =\left(\left(c_{i} t+r-\alpha\right)\left(2 c_{i}\left(c_{i}+c\right)\right)^{-1}\right.
\end{aligned}
$$

Hence, using $\lambda \geq c_{i}(t-s)$, we have

$$
\begin{aligned}
(1+t-s)\left(1+c_{i} s+\lambda\right)^{\mu} & \geq(1+(t-s))\left(1+c_{i} s+\lambda\right)\left(1+c_{i}(t-s)\right)^{\mu-1} \\
& \geq(1+\alpha)\left(1+c_{i} t+r-\alpha\right)^{\mu}
\end{aligned}
$$

Making use of the formula (10.15), we have

$$
\begin{gathered}
\int_{D_{2}^{(i)}}(1+t-s)^{-1}\left(1+\left|c_{j} s-\lambda\right|\right)^{-\mu}\left(1+c_{i} s+\lambda\right)^{-\mu} d \lambda d s \\
\leq C\left(1+\left|c_{i} t-r\right|\right)^{-1} \int_{\left|c_{i} t-r\right|}^{c_{i} t+r}\left(1+c_{i} t+r-\alpha\right)^{-\mu} d \alpha \int_{\alpha_{0}}^{\alpha}(1+|\beta|)^{-\mu} d \beta
\end{gathered}
$$


which is estimated by the right hand side of (10.26). We next treat the second integral of (10.26) which will be denoted by $I_{0}$. If $r \geq 2 c_{1} t, z_{\mu, \mu}^{(j)}(s, \lambda) \geq C(1+r)^{2 \mu}$. Then we have

$$
\begin{aligned}
I_{0} & \leq C(1+r)^{-2 \mu} \int_{0}^{t} d s \int_{\lambda_{1}^{(i)}}^{\lambda_{2}^{(i)}}\left\{\left(\lambda-\lambda_{1}^{(i)}\right)\left(\lambda_{2}^{(i)}-\lambda\right)\right\}^{-1 / 2} d \lambda \\
& =C \pi t(1+r)^{-2 \mu} \leq C(1+t+r)^{-1} .
\end{aligned}
$$

If $r \leq 2 c_{1} t$ and $0<c_{i} s<c_{i} t-r$, then we have

$$
\left(\lambda-\lambda_{1}^{(i)}\right)\left(\lambda_{2}^{(i)}-\lambda\right)=\left(\alpha-c_{i} t+r\right)\left(c_{i} t+r-\alpha\right) .
$$

Making use of the formula (10.15),

$$
\begin{aligned}
I_{0} \leq & C \int_{c_{i} t-r}^{c_{i} t+r}(1+\alpha)^{-\mu}\left\{\left(\alpha-c_{i} t+r\right)\left(c_{i} t+r-\alpha\right)\right\}^{-1 / 2} d \alpha \times \\
& \times \int_{\alpha_{0}}^{\alpha}(1+|\beta|)^{-\mu} d \beta \leq C \pi\left(1+\left|c_{i} t-r\right|\right)^{-\mu} \Phi_{\mu}(t) .
\end{aligned}
$$

If $r \leq 2 c_{1} t$ and $\left(c_{i} t-r\right)_{+}<c_{i} s<c_{i} t$, we have

$$
\begin{aligned}
\lambda-\lambda_{1}^{(i)} & =\lambda+c_{i}(t-s)-r \\
& =2 c_{i}\left(\beta-\alpha_{0}\right)\left(c_{i}+c\right)^{-1} .
\end{aligned}
$$

Making use of the formula (10.15),

$$
I_{0} \leq C \int_{\left|c_{i} t-r\right|}^{c_{i} t+r}(1+\alpha)^{-\mu}\left(1+c_{i} l+r-\alpha\right)^{-1 / 2} d \alpha \int_{\alpha_{0}}^{\alpha}\left(1+\beta-\alpha_{0}\right)^{-1 / 2}(1+|\beta|)^{-\mu} d \beta
$$

We will prove

$$
\begin{aligned}
& \int_{\alpha_{0}}^{\alpha}\left(1+\beta-\alpha_{0}\right)^{-1 / 2}(1+|\beta|)^{-\mu} d \beta \\
\leq & C\left\{\left(1+\left|\alpha_{0}\right|\right)^{1 / 2-\mu}+\chi\left(\alpha_{0}\right) \Phi_{\mu}(t)\left(1+\left|\alpha_{0}\right|\right)^{-1 / 2}\right\},
\end{aligned}
$$

where $\chi$ is the characteristic function of the interval $(-\infty, 0)$. In fact, if $\alpha_{0} \geq 0$, then integration by parts yields

$$
\begin{aligned}
& \int_{\alpha_{0}}^{\alpha}\left(1+\beta-\alpha_{0}\right)^{-1 / 2}(1+\beta)^{-\mu} d \beta \\
\leq & 2(1+\alpha)^{1 / 2-\mu}+2 \mu \int_{\alpha_{0}}^{\alpha}(1+\beta)^{-1 / 2-\mu} d \beta \\
\leq & C\left(1+\alpha_{0}\right)^{1 / 2-\mu} .
\end{aligned}
$$


Here we have used (10.17). If $\alpha_{0}<0$, then $1+\beta-\alpha_{0}<1-\beta$ for $\alpha_{0}<\beta<\alpha_{0} / 2$ and $1+\beta-\alpha_{0}>-\alpha_{0} / 2$ for $\beta>\alpha_{0} / 2$. Deviding the integral into two intervals, we obtain (10.30). Thus, we find from (10.29) and (10.30) that

$$
\begin{aligned}
I_{0} & \leq C\left(1+\left|c_{i} t+r\right|\right)^{-1} \Phi_{\mu}(t) \times \\
& \times \int_{\left|c_{i} t-r\right|}^{c_{i} t+r}(1+\alpha)^{-\mu+1}\left(1+c_{i} t+r-\alpha\right)^{-1 / 2}\left(1+\left|\alpha_{0}\right|\right)^{-1 / 2} d \alpha \\
& \leq C\left(1+\left|c_{i} t+r\right|\right)^{-1}\left(\Phi_{\mu}(t)\right)^{2} .
\end{aligned}
$$

Therefore, we have proved the assertion (10.26).

The rest of this section is devoted to the proof of Proposition 6.2 (ii) (iii) for

$$
\begin{aligned}
& \int_{0}^{t}(t-s) d s \int_{c_{2}(t-s)}^{c_{1}(t-s)} \tau^{-2} J_{a}(\partial F(s, y) ; x, \tau) d \tau \\
= & \frac{1}{4 \pi r} \int_{0}^{t}(t-s) d s \int_{D} \tau^{-2} \lambda d \lambda d \tau \int_{0}^{2 \pi} \Xi^{a} \partial F(s, \lambda \Theta) d \varphi .
\end{aligned}
$$

where

$$
D=\left\{(\lambda, \tau): c_{2}(t-s) \leq \tau \leq c_{1}(t-s),|r-\tau| \leq \lambda \leq r+\tau\right\}
$$

We divide the integral domain $D$ into $D_{1}$ and $D_{2}$ :

$$
\begin{aligned}
D_{1}= & \left\{(\lambda, \tau): c_{2}(t-s) \leq \tau \leq c_{1}(t-s),|r-\tau| \leq \lambda \leq|r-\tau|+\delta\right. \\
& \quad \text { or } r+\tau-\delta \leq \lambda \leq r+\tau\}, \\
D_{2}= & D \backslash D_{1}, \quad \delta=\min (1, r) .
\end{aligned}
$$


Making use of (10.19) and (10.31), the integration by parts yields

$$
\begin{aligned}
& 4 \pi r \int_{0}^{t}(t-s) d s \int_{c_{2}(t-s)}^{c_{1}(t-s)} \tau^{-2} J_{a}(\nabla F(s, y) ; x, \tau) d \tau \\
= & \int_{0}^{t}(t-s) d s \int_{D_{1}} \tau^{-2} \lambda d \lambda d \tau \int_{0}^{2 \pi} \Xi^{a} \nabla F(s, \lambda \Theta) d \varphi \\
& +\int_{0}^{t}(t-s) d s \int_{c_{2}(t-s)}^{c_{1}(t-s)} \tau^{-2}\left[\lambda \Theta \int_{0}^{2 \pi} \Xi^{a} F(s, \lambda \Theta) d \varphi\right]_{\substack{\lambda=|r-\tau|+\delta \\
\lambda=r}}^{\lambda=r+\delta} d \tau
\end{aligned}
$$

$(10.32)$

$$
\begin{aligned}
& +\int_{0}^{t}(t-s) d s \int_{D_{2}} \tau^{-2} \partial_{\lambda}\left(\lambda \Theta \Xi^{a}\right) d \lambda d \tau \int_{0}^{2 \pi} F(s, \lambda \Theta) d \varphi \\
& +\int_{0}^{t}(t-s) d s \int_{D_{2}} \tau^{-2} d \lambda d \tau \int_{0}^{2 \pi} \Xi^{a}\left[\Theta\left\{\partial_{\lambda} \Theta \cdot(\Theta \wedge \Omega F(s, \lambda \Theta))\right\}\right. \\
& \left.\quad-\lambda^{-1} \Theta \wedge \Omega F(s, \lambda \Theta)\right] d \varphi
\end{aligned}
$$

$$
\begin{aligned}
& 4 \pi r \int_{0}^{t}(t-s) d s \int_{c_{2}(t-s)}^{c_{1}(t-s)} \tau^{-2} J_{a}\left(\partial_{t} F(s, y) ; x, \tau\right) d \tau \\
& =\int_{0}^{t}(t-s) d s \int_{D_{1}} \tau^{-2} \lambda d \lambda d \tau \int_{0}^{2 \pi} \Xi^{a} \partial_{t} F(s, \lambda \Theta) d \varphi \\
& +t \int_{c_{2} t}^{c_{1} t} \tau^{-2} d \tau \int_{|r-\tau|+\delta}^{r+\tau-\delta} \lambda d \lambda \int_{0}^{2 \pi} \Xi^{a} F(s, \lambda \Theta) d \varphi \\
& +\sum_{i=1}^{2}(-1)^{i} \int_{0}^{t} C_{i}^{-2}(t-s)^{-1} J_{a}\left(F(s, y) ; x, c_{i}(t-s)\right) d s \\
& -\int_{0}^{t} \cdot d s \int_{D_{2}} \tau^{-2} d \lambda d \lambda d \tau \int_{0}^{2 \pi} \Xi^{a} F(s, \lambda \Theta) d \varphi .
\end{aligned}
$$

The third integral in the right hand side of (10.33) has been treated in (10.22), (10.23) and is estimated by the first integral of (10.26). Therefore, we find from (6.14), (10.10), (10.32) and (10.33) that Proposition 6.2 (ii) (iii) follows from the following estimates:

$$
I_{0}^{k} \leq C w(t, r)^{-1}\left(\Phi_{\mu}(t)\right)^{2}, \quad k=0,1, \cdots 8
$$


where

$$
\begin{aligned}
& I_{0}^{0}=r^{-1} \int_{0}^{t}(t-s) d s \int_{D_{1}} \tau^{-2} z_{\mu, \mu}^{(j)}(s, \lambda) d \lambda d \tau \\
& I_{0}^{1}=(1+r)^{-1} \int_{0}^{t}(t-s) d s \int_{c_{2}(t-s)}^{c_{1}(t-s)} \tau^{-2} z_{\mu, \mu}^{(j)}(s, r+\tau)^{-1} d \tau, \\
& I_{0}^{2}=(1+r)^{-1} \int_{0}^{t}(t-s) d s \int_{c_{2}(t-s)}^{c_{1}(t-s)} \tau^{-2} z_{\mu, \mu}^{(j)}(s,|r-\tau|)^{-1} d \tau, \\
& I_{0}^{3}=(1+r)^{-1} \int_{0}^{t}(t-s) d s \int_{D_{2}} \tau^{-2} \lambda^{-1} z_{\mu, \mu}^{(j)}(s, \lambda) d \lambda d \tau, \\
& I_{0}^{4}=(1+r)^{-1} \int_{0}^{t}(t-s) d s \int_{D_{2}} \tau^{-3} z_{\mu, \mu}^{(j)}(s, \lambda) d \lambda d \tau, \\
& I_{0}^{5}=(1+r)^{-1} \int_{0}^{t}(t-s) d s \int_{D_{2}} \tau^{-2}\left|\partial_{\lambda} \Theta\right| z_{\mu, \mu}^{(j)}(s, \lambda)^{-1} d \lambda d \tau, \\
& I_{0}^{6}=(1+r)^{-1} \int_{0}^{t}(t-s) d s \int_{D_{2}} \tau^{-3} \lambda\left|\partial_{\lambda} \Theta\right| z_{\mu, \mu}^{(j)}(s, \lambda) d \lambda d \tau \\
& I_{0}^{7}=(1+r)^{-1} t \int_{c_{2} t}^{c_{1} t}(\tau+1)^{-2} d \tau \int_{|r-\tau|}^{r+\tau} z_{\mu, \mu}^{(j)}(0, \lambda)^{-1} d \lambda, \\
& I_{0}^{8}=(1+r)^{-1} \int_{0}^{t} d s \int_{D_{2}} \tau^{-2} z_{\mu, \mu}^{(j)}(s, \lambda)^{-1} d \lambda d \tau \\
& (j=0,1,2) \text {. }
\end{aligned}
$$

We first prove (10.34) for $I_{0}^{k}(k=1.7)$. Since $1+c_{1} s+r+\tau \geq 1+c_{2} t+r$ for $\tau \geq c_{2}(t-s)$, we have

$$
\begin{aligned}
& I_{0}^{1} \leq(r+1)^{-1}\left(1+c_{2} t+r\right)^{-\mu} \times \\
& \times \int_{0}^{t}(t-s) d s \int_{c_{2}(t-s)}^{c_{1}(t-s)} \tau^{-2}\left(1+\left|c_{j} s-\tau-r\right|\right)^{-\mu} d \tau .
\end{aligned}
$$

Integration by parts in $\tau$ yields

$$
I_{0}^{1} \leq C(r+1)^{-1}\left(1+c_{2} t+r\right)^{-\mu} \Phi_{\mu}(t) .
$$

Since $z_{\mu, \mu}^{(j)}(0, \lambda)=(1+\lambda)^{2 \mu}$, we have

$$
\begin{aligned}
I_{0}^{7} & \leq C(1+r)^{-1}(1+t)^{-1} \int_{c_{2} t}^{c_{1} t}(1+|r-\tau|)^{-2 \mu+1} d \tau \\
& \leq C(1+r)^{-1}(1+t+r)^{-1} \Phi_{\mu}(t) .
\end{aligned}
$$


Moreover, $I_{0}^{8}$ is equivalent to $I_{0}^{4}$ and

$$
I_{0}^{0} \leq C\left(I_{0}^{1}+I_{0}^{2}\right)
$$

because $r^{-1} \delta \leq 2(r+1)^{-1}$. Thus, we will prove (10.34) for $I_{0}^{k}(2 \leq k \leq 6)$ by separating four cases.

Case 1. $r \geq 2 c_{1} t$.

In this case, it holds that $1+\left|c_{j} s-\lambda\right| \geq r / 2(j=0,1,2)$ and $1+c_{1} s+\lambda \geq r / 2$ in the domain of integration. Then we easily see

$$
I_{0}^{k} \leq C t(1+r)^{-1-2 \mu} \leq C(1+r)^{-1}(1+t+r)^{-1}, \quad k=2,3 .
$$

By the definition of $I_{0}^{4}$, we easily see

$$
\begin{aligned}
I_{0}^{4} & \leq C(1+r)^{-1-2 \mu} \int_{0}^{t} d s \int_{c_{2}(t-s)}^{c_{1}(t-s)}(1+\tau)^{-2} d \tau \\
& \leq C(1+r)^{-1}(1+t+r)^{-1} \Phi_{\mu}(t) .
\end{aligned}
$$

From (10.24),

$$
\left|\partial_{\lambda} \Theta\right| \leq 2 \tau h(\lambda, \tau)^{-1 / 2} \leq 2\{(\tau-|\dot{r}-\lambda|)(r+\lambda-\tau)\}^{-1 / 2}
$$

and

$$
\tau^{-1} \lambda\left|\partial_{\lambda} \Theta\right| \leq 2 \lambda h(\lambda, \tau)^{-1 / 2} \leq 2\{(\lambda-|r-\tau|)(r+\tau-\lambda)\}^{-1 / 2}
$$

Hence, we find from (10.36) that

$$
\begin{aligned}
I_{0}^{6} & \leq C \pi(1+r)^{-1-2 \mu} \int_{0}^{t}(t-s) d s \int_{c_{2}(t-s)}^{c_{1}(t-s)} \tau^{-2} d \tau \\
& \leq C(1+r)^{-1}(1+t+r)^{-1} .
\end{aligned}
$$

Extending the domain $D_{2}$ of $(\lambda, \tau)$-integration to $\left\{(\lambda, \tau) ; \lambda_{1}^{(1)} \leq \lambda \leq \lambda_{2}^{(1)},|\lambda-r| \leq \tau\right.$ $\left.\leq c_{1}(t-s)\right\}$ and inverting the order of $(\lambda, \tau)$-integration, we find from (10.35) that

$$
\begin{aligned}
I_{0}^{5} & \leq C \pi(1+r)^{-1-2 \mu} \int_{0}^{t}(1+t-s)^{-1} d s \int_{\lambda_{1}^{(1)}}^{\lambda_{2}^{(1)}} d \lambda \\
& \leq C(1+r)^{-1}(1+t+r)^{-1} \Phi_{\mu}(t)
\end{aligned}
$$


Case 2. $c_{1} t \leq r \leq 2 c_{1} t$ or $c_{2} t \leq r \leq c_{1} t, c_{1}(t-s)<r$.

Since $r \geq \tau$ in this case, it holds that

$$
I_{0}^{2} \leq C(1+r)^{-1} \int_{\left(c_{1} t-r\right)_{+} / c_{1}}^{t}(t-s) d s \int_{c_{2}(t-s)}^{c_{1}(t-s)} \tau^{-2} z_{\mu, \mu}^{(j)}(s, r-\tau) d \tau
$$

In the domain of integration,

$$
1+c_{1} s+\lambda \geq 1+c_{1} s+r-\tau \geq 1+\left|c_{1} t-r\right|
$$

In fact,

$$
\begin{aligned}
& c_{1} s+r-\tau \geq c_{1} s+r-c_{1}(t-s)=r-c_{1} t+2 c_{1} s \\
& \geq \begin{cases}r-c_{1} t & \text { for } r \geq c_{1} t, \\
r-c_{1} t+2\left(c_{1} t-r\right) & \text { for } r \leq c_{1} t, c_{1}(t-s) \leq r .\end{cases}
\end{aligned}
$$

Hence, making use of (10.37), we have

$$
\begin{aligned}
& (1+r)\left(1+c_{1} t-r \mid\right)^{\mu} I_{0}^{2} \\
\leq & C \int_{0}^{t}(t-s) d s \int_{c_{2}(t-s)}^{c_{1}(t-s)} \tau^{-2}\left(1+\left|c_{j} s-r+\tau\right|\right)^{-\mu} d \tau \\
\leq & C \Phi_{\mu}(t) .
\end{aligned}
$$

Extending the domain of $(\lambda, \tau)$-integration in $I_{0}^{3}$ to $\left[\lambda_{1}^{(1)}, \lambda_{2}^{(1)}\right] \times\left[c_{2}(t-s), c_{1}(t-s)\right]$,

$$
\begin{aligned}
& (1+r) I_{0}^{3} \\
\leq & C \int_{0}^{t} d s \int_{\lambda_{1}^{(1)}}^{\lambda_{2}^{(1)}}(\lambda+1)^{-1}\left(1+\left|c_{j} s-\lambda\right|\right)^{-\mu}\left(1+c_{1} s+\lambda\right)^{-\mu} d \lambda .
\end{aligned}
$$

For $j=0$, the change of variables (10.14) with $c=0$ yields

$$
\begin{aligned}
I_{0}^{3} & \leq C(1+r)^{-1} \int_{\left|c_{1} t-r\right|}^{c_{1} t+r}(1+\alpha)^{-\mu} d \alpha \int_{\alpha_{0}}^{\alpha}(1+\beta)^{-1-\mu} d \beta \\
& \leq C(1+r)^{-1}\left(1+\left|c_{1} t-r\right|\right)^{-\mu} \int_{\left|c_{1} t-r\right|}^{c_{1} t+r}\left(1+\left|\alpha_{0}\right|\right)^{-\mu} d \beta \\
& \leq C(1+r)^{-1}\left(1+\left|c_{1} t-r\right|\right)^{-\mu} \Phi_{\mu}(t) .
\end{aligned}
$$


Since

$$
\begin{aligned}
& (1+\lambda)^{-1}\left(1+\left|c_{j} s-\lambda\right|\right)^{-\mu} \\
& \leq \begin{cases}C(1+\lambda)^{-1}\left(1+c_{1} s+\lambda\right)^{-\mu} & \text { for } \lambda \leq c_{j} s / 2 \\
C\left(1+\left|c_{j} s-\lambda\right|\right)^{-\mu}\left(1+c_{1} s+\lambda\right)^{-1} & \text { for } \lambda \geq c_{j} s / 2\end{cases}
\end{aligned}
$$

for $j=1,2$, it holds that

$$
(1+\lambda)^{-1} z_{\mu, \mu}^{(j)}(s, \lambda) \leq C\left\{(1+\lambda)^{-\mu}+\left(1+\left|c_{j} s-\lambda\right|\right)^{-\mu}\right\}\left(1+c_{1} s+\lambda\right)^{-1-\mu}
$$

which implies by the change of variables (10.14)

$$
I_{0}^{3} \leq C(1+r)^{-1}\left(1+\left|c_{1} t-r\right|\right)^{-\mu} \Phi_{\mu}(t) .
$$

Extending the domain of $(\lambda, \tau)$-integration to $\left[\lambda_{1}^{(1)}, \lambda_{2}^{(1)}\right] \times\left[c_{2}(t-s), c_{1}(t-s)\right]$, we have

$$
\begin{aligned}
& (1+r) I_{0}^{4} \\
\leq & C \int_{0}^{t} d s \int_{c_{2}(t-s)}^{c_{1}(t-s)}(1+\tau)^{-2} d \tau \int_{\lambda_{1}^{(1)}}^{\lambda_{2}^{(1)}} z_{\mu, \mu}^{(j)}(s, \lambda) d \lambda \\
\leq & C \int_{0}^{t}(1+t-s)^{-1} d s \int_{\lambda_{1}^{(1)}}^{\lambda_{2}^{(1)}}\left(1+\left|c_{j} s-\lambda\right|\right)^{-\mu}\left(1+c_{1} s+\lambda\right)^{-\mu} d \lambda .
\end{aligned}
$$

which is the same integral as (10.28) with $i=1$. Hence,

$$
I_{0}^{4} \leq C(1+r)^{-1}\left(1+c_{1} t-r\right)^{-1}\left(\Phi_{\mu}(t)\right)^{2}
$$

Extending the domain $D_{2}$ of $(\lambda, \tau)$-integration to $\left\{(\lambda, \tau) ; \lambda_{1}^{(1)} \leq \lambda \leq \lambda_{2}^{(1)},|\lambda-r| \leq\right.$ $\left.\tau \leq c_{1}(t-s)\right\}$ and inverting the order of $(\lambda, \tau)$-integration, we find from (10.35) that

$$
\begin{aligned}
& (1+r) I_{0}^{5} \\
\leq & C \int_{0}^{t}(1+t-s)^{-1} d s \int_{\lambda_{1}^{(1)}}^{\lambda_{2}^{(1)}}\left(1+\left|c_{j} s-\lambda\right|\right)^{-\mu}\left(1+c_{1} s+\lambda\right)^{-\mu} d \lambda
\end{aligned}
$$

which is the same integral as (10.28) with $i=1$. Hence,

$$
I_{0}^{5} \leq C(1+r)^{-1}\left(1+\left|c_{1} t-r\right|\right)^{-1}\left(\Phi_{\mu}(t)\right)^{2} .
$$


Extending the domain $D_{2}$ of $(\lambda, \tau)$-integration to $\left\{(\lambda, \tau), \lambda_{1}^{(1)} \leq \lambda_{2}^{(1)}, 1+|\lambda-r| \leq\right.$ $\left.\tau \leq c_{1}(t-s)\right\}$, we find from (10.36) with $\tau \leq r$ that

$$
\begin{aligned}
& (1+r) I_{0}^{6} \\
\leq & C \int_{0}^{t} d s \int_{\lambda_{1}^{(1)}}^{\lambda_{2}^{(1)}} z_{\mu, \mu}^{(j)}(s, \lambda) d \lambda \int_{1+|\lambda-r|}^{c_{1}(t-s)} \tau^{-1}\left\{\tau^{2}-(\lambda-r)^{2}\right\}^{-1 / 2} d \tau .
\end{aligned}
$$

Since

$$
\int \frac{d \tau}{\tau \sqrt{\tau^{2}-k^{2}}}=\frac{2}{|k|} \operatorname{Tan}^{-1} \frac{\tau+\sqrt{\tau^{2}-k^{2}}}{|k|}
$$

it holds that

$$
\begin{aligned}
& \int_{1+|\lambda-r|}^{c_{1}(t-s)} \tau^{-1}\left\{\tau^{2}-(\lambda-r)^{2}\right\}^{-1 / 2} d \tau \\
\leq & \int_{1+|\lambda-r|}^{c_{1}(t-s)} \tau^{-1}\left\{\tau^{2}-(|\lambda+r|+1)^{2}\right\}^{-1 / 2} d \tau \\
\leq & \pi(1+|\lambda-r|)^{-1} .
\end{aligned}
$$

Hence, we find from (10.37) and (10.39) that

$$
I_{0}^{6} \leq C(1+r)^{-1}\left(1+\left|c_{i} t-r\right|\right)^{-1} I_{0,1}^{6}
$$

where

$$
I_{0,1}^{6}=\int_{0}^{t} d s \int_{r-c_{1}(t-s)}^{r+c_{1}(t-s)}\left(1+c_{1} s+\lambda\right)^{1-\mu}\left(1+\left|c_{j} s-\lambda\right|\right)^{-\mu}(1+|\lambda-r|)^{-1} d \lambda
$$

Since

$$
\begin{aligned}
I_{0,1}^{6} & \leq \int_{r}^{c_{1} t+r}(1+\lambda)^{1-\mu}(1+\lambda-r)^{-1} d \lambda \int_{0}^{\left(c_{1} t+r-\lambda\right) / c_{1}}\left(1+\left|c_{j} s-\lambda\right|\right)^{-\mu} d s \\
& +\int_{c_{1} t-r}^{r}(1+\lambda)^{1-\mu}(1+\lambda-r)^{-1} d \lambda \int_{0}^{\left(r-c_{1} t-\lambda\right) / c_{1}}\left(1+\left|c_{j} s-\lambda\right|\right)^{-\mu} d s
\end{aligned}
$$

we conclude that

$$
I_{0}^{6} \leq C(1+r)^{-1}\left(1+\left|c_{1} t-r\right|\right)^{-1}\left(\Phi_{\mu}(t)\right)^{2}
$$


Case 3. $c_{2} t \leq r \leq c_{1} t, c_{1}(t-s)>r$ or $r \leq c_{2} t, c_{2}(t-s)<r$.

In this case,

$$
I_{0}^{2} \leq C(1+r)^{-1} \int_{\left(c_{2} t-r\right)_{+} / c_{2}}^{\left(c_{1} t-r\right) / c_{1}}(t-s) d s \int_{c_{2}(t-s)}^{c_{1}(t-s)} \tau^{-2} z_{\mu, \mu}^{(j)}(s,|\tau-r|)^{-1} d \tau .
$$

In the case where $c_{2} t \leq r \leq c_{1} t$,

$$
\tau \geq c_{2}(t-s) \geq c_{2} c_{1}^{-1} r \geq C(r+t)
$$

Hence,

$$
\begin{aligned}
& (1+r)(1+t+r) I_{0}^{2} \\
\leq & C \int_{\left(c_{2} t-r\right)_{+} / c_{2}}^{\left(c_{1} t-r\right) / c_{1}} d s \int_{\left(c_{2} t-s\right)}^{c_{1}(t-s)}\left(1+|r-\tau|+c_{1} s\right)^{-\mu}\left(1+\left|c_{j} s-\right| r-\tau||\right)^{-\mu} d \tau \\
\leq & C \int_{0}^{t}\left(1+c_{1} s\right)^{-\mu} d s \int_{c_{2}(t-s)}^{c_{1}(t-s)}\left(1+\left|c_{j} s-\right| r-\tau \mid\right)^{-\mu} d \tau \\
\leq & C\left(\Phi_{\mu}(t)\right)^{2} .
\end{aligned}
$$

In the case where $r \leq c_{2} t, c_{2}(t-s)<r$,

$$
c_{1} s \geq c_{1} c_{2}^{-1}\left(c_{2} t-r\right)
$$

Hence, the integration by parts yield

$$
\begin{aligned}
(1+r) I_{0}^{2} & \leq C\left(1+c_{2} t-r\right)^{-\mu} \int_{0}^{t}(t-s) d s \int_{c_{2}(t-s)}^{c_{1}(t-s)} \tau^{-2}\left(1+\left|c_{j} s-\right| r-\tau \mid\right)^{-\mu} d \tau \\
& \leq C\left(1+c_{2} t-r\right)^{-\mu} \Phi_{\mu}(t) .
\end{aligned}
$$

Dividing the integral domain $D_{2}$ into $D_{2} \cap\{\tau \leq r\}$ and $D_{2} \cap\{\tau \geq r\}$ and inverting the order of $(\lambda, \tau)$-integration, one can verify that

$$
I_{0}^{3} \leq C(1+r)^{-1} \sum_{i=1}^{5} I_{0, i}^{3}
$$


where

$$
\begin{aligned}
I_{0, i}^{3} & =\int_{0}^{t} d s \int_{\lambda_{1}^{(1)}}^{\lambda_{2}^{(i)}}(\lambda+1)^{-1}\left(1+c_{i} s+\lambda\right)^{-\mu}\left(1+\left|c_{j} s-\lambda\right|\right)^{-\mu} d \lambda \quad(i=1,2) \\
I_{0,3}^{3} & =\int_{\left(c_{2} t-r\right)_{+} / c_{2}}^{\left(c_{1} t-r\right) / c_{1}}(t-s) d s \int_{0}^{\lambda_{1}^{(1)}} \lambda^{-1} z_{\mu, \mu}^{(j)}(s, \lambda) d \lambda \int_{r}^{r+\lambda+1} \tau^{-2} d \tau \\
I_{0,4}^{3} & =\int_{\left(c_{2} t-r\right)_{+} / c_{2}}^{\left(c_{1} t-r\right) / c_{1}}(t-s) d s \int_{0}^{\lambda_{1}^{(2)}} \lambda^{-1} z_{\mu, \mu}^{(j)}(s, \lambda) d \lambda \int_{1+r-\lambda}^{r} \tau^{-2} d \tau \\
I_{0,5}^{3} & =\int_{\left(c_{2} t-r\right)_{+} / c_{2}}^{\left(c_{1} t-r\right) / c_{1}}(t-s) d s \int_{\lambda_{2}^{(2)}}^{2 r} \lambda^{-1} z_{\mu, \mu}^{(j)}(s, \lambda) d \lambda \int_{1+\lambda-r}^{r} \tau^{-2} d \tau .
\end{aligned}
$$

Since $I_{0, i}^{3}(i=1,2)$ is the same integral as one in $(10.38)$, we obtain

$$
l_{0, i}^{3} \leq C(1+r)^{-1}\left(1+\left|c_{i} t-r\right|\right)^{-\mu} \Phi_{\mu}(t), \quad i=1,2 .
$$

Each $\tau$-integral is estimated by

$$
C \lambda r^{-1}(1+t-s)^{-1}
$$

Hence, in the case where $c_{2} t \leq r \leq c_{1} t$, it follows from (10.40) that

$$
\begin{aligned}
I_{0, k}^{3} & \leq C(1+t+r)^{-1} \int_{0}^{t}\left(1+c_{1} s\right)^{-\mu} d s \int_{0}^{\lambda_{1}^{(k-2)}}\left(1+\left|c_{j} s-\lambda\right|\right)^{-\mu} d \lambda \\
& \leq C(1+t+r)^{-1}\left(\Phi_{\mu}(t)\right)^{2}, \quad k=3,4
\end{aligned}
$$

and

$$
\begin{aligned}
I_{0,5}^{3} & \leq C(1+t+r)^{-1} \int_{0}^{t} d s \int_{\lambda_{2}^{(2)}}^{2 r}\left(1+c_{2} s+\lambda\right)^{-1}\left(1+\left|c_{j} s-\lambda\right|\right)^{-\mu} d \lambda \\
& \leq C(1+t+r)^{-1} \Phi_{\mu}(t)
\end{aligned}
$$

here we have used that $1+c_{2} s+\lambda \geq \lambda_{2}^{(2)} \geq r \geq c_{2} t$. In the case where $r \leq c_{2} t$, $c_{2}(t-s) \leq r$, it follows from $(10.39),(10.40)$ that

$$
\begin{aligned}
I_{0, k}^{3} & \leq C r^{-1}\left(1+c_{2} t-r\right)^{-1} \int_{\left(c_{2} t-r\right) / c_{2}}^{\left(c_{1} t-r\right) / c_{1}} d s \int_{0}^{\lambda_{1}^{(k-2)}}\left(1+c_{j} s-\lambda\right)^{-\mu} d \lambda \\
& \leq C\left(1+c_{2} t-r\right)^{-1} \Phi_{\mu}(t), \quad k=3,4
\end{aligned}
$$


and, in the same way,

$$
I_{0,5}^{3} \leq C\left(1+c_{2} t-r\right)^{-1} \Phi_{\mu}(t) .
$$

If $\tau \geq \lambda, I_{0}^{4} \leq I_{0}^{3}$. Then we can assume in the treatment of $I_{0}^{4}$ that $\lambda \geq \tau$. Set $\bar{D}_{2}=D_{2} \cap\{\lambda \geq \tau\}$. Then,

$$
I_{0}^{4} \leq C(1+r)^{-1} \int_{\left(c_{2} t-r\right)_{+} / c_{2}}^{\left(c_{1} t-r\right) / c_{1}} d s \int_{\bar{D}_{2}} \tau^{-2} z_{\mu, \mu}^{(j)}(s, \lambda) d \lambda d \tau .
$$

Since $r-c_{2}(t-s) \geq 2^{-1} r$ and $t-s \geq c_{1}^{-1} r$, inverting the order of $(\lambda, \tau)$-integration yields

$$
I_{0}^{4} \leq C(1+r)^{-1} \sum_{i=1}^{3} I_{0, i}^{4}
$$

where

$$
\begin{aligned}
& I_{0, i}^{4}=(1+r)^{-1} \int_{0}^{t} d s \int_{\lambda_{1}^{(i)}}^{\lambda_{2}^{(i)}}\left(1+c_{i} s+\lambda\right)^{-\mu}\left(1+\left|c_{j} s-\lambda\right|\right)^{-\mu} d \lambda \quad(i=1,2) \\
& I_{0,3}^{4}=(1+r)^{-1} \int_{\left(c_{2} t-r\right)_{+} / c_{2}}^{\left(c_{1} t-r\right) / c_{1}} d s \int_{\lambda_{2}^{(2)}}^{2 r}\left(1+c_{1} s+\lambda\right)^{-\mu}\left(1+\left|c_{j} s-\lambda\right|\right)^{-\mu} d \lambda .
\end{aligned}
$$

Making use of the change of variables (10.14),

$$
\begin{aligned}
I_{0, i}^{4} & \leq(1+r)^{-1} \int_{\left|c_{i} t-r\right|}^{c_{i} t+r}(1+\alpha)^{-\mu} d \alpha \int_{\alpha_{0}}^{\alpha}(1+|\beta|)^{-\mu} d \beta \\
& \leq\left(1+\left|c_{i} t-r\right|\right)^{-\mu} \Phi_{\mu}(t) .
\end{aligned}
$$

Since

$$
1+c_{1} s+\lambda \geq \begin{cases}1+r \geq C(1+t+r) & \text { for } c_{2} t \leq r \leq c_{1} t \\ 1+c_{2} t-r & \text { for } r \leq c_{2} t\end{cases}
$$

we conclude that

$$
I_{0,3}^{4} \leq \Phi_{\mu}(t) \begin{cases}(1+t+r)^{-1} & \text { for } c_{2} t \leq r \leq c_{1} t \\ \left(1+c_{2} t-r\right)^{-1} & \text { for } r \leq c_{2} t\end{cases}
$$

Extending the domain $D_{2}$ of $(\lambda, \tau)$-integration to $\left\{(\lambda, \tau): 0<\lambda<\lambda_{2}^{(1)},|\lambda-r| \leq\right.$ 
$\tau<\lambda+r\}$, it follows from (10.35) that

$$
\begin{aligned}
& (1+r) I_{0}^{5} \\
\leq & C \pi \int_{\left(c_{2} t-r\right)_{+} / c_{2}}^{\left(c_{1} t-r\right) / c_{1}}(1+t-s) d s \int_{0}^{\lambda_{2}^{(1)}} z_{\mu, \mu}^{(j)}(s, \lambda)^{-1} d \lambda \\
\leq & C \sum_{i=1}^{2} \int_{0}^{t}(1+t-s)^{-1} d s \int_{\lambda_{1}^{(i)}}^{\lambda_{2}^{(i)}}\left(1+c_{i} s+\lambda\right)^{-\mu}\left(1+\left|c_{j} s-\lambda\right|\right)^{-\mu} d \lambda \\
(10.41) & +C \int_{\left(c_{2} t-r\right)_{+} / c_{2}}^{\left(c_{1} t-r\right) / c_{1}}(1+t-s)^{-1} d s \int_{0}^{\lambda_{1}^{(2)}}\left(1+c_{1} s+\lambda\right)^{-\mu}\left(1+\left|c_{j} s-\lambda\right|\right)^{-\mu} d \lambda .
\end{aligned}
$$

The first integral of $(10.41)$ is same as $(10.28)$ and then estimated by

$$
C\left(1+\left|c_{i} t-r\right|\right)^{-1}\left(\Phi_{\mu}(t)\right)^{2}
$$

In the case where $c_{2} t \leq r \leq c_{1} t$, the second integral of (10.41) is estimated by

$$
\begin{aligned}
& C(1+t+r)^{-1} \int_{0}^{t}\left(1+c_{1} s\right)^{-\mu} d s \int_{0}^{\lambda_{1}^{(2)}}\left(1+\left|c_{j} s-\lambda\right|\right)^{-\mu} d \lambda \\
\leq & C(1+t+r)^{-1}\left(\Phi_{\mu}(t)\right)^{2} .
\end{aligned}
$$

In the case where $r \leq c_{2} t, c_{2}(t-s)<r$, the second integral of $(10.41)$ is estimated

$$
\begin{aligned}
& C(1+r)^{-1}\left(1+c_{2} t-r\right)^{-\mu} \int_{\left(c_{2} t-r\right) / c_{2}}^{\left(c_{1} t-r\right) / c_{1}} d s \int_{0}^{\lambda_{1}^{(2)}}\left(1+\left|c_{j} s-\lambda\right|\right)^{-\mu} d \lambda \\
\leq & C\left(1+c_{2} t-r\right)^{-\mu} \Phi_{\mu}(t) .
\end{aligned}
$$

If $\tau \geq \lambda, I_{0}^{6} \leq I_{0}^{5}$. Then we can assume that $\lambda \geq \tau$. By (10.36),

$$
\left|\tau^{-1} \lambda \partial_{\lambda} \Theta\right| \leq \begin{cases}\left(\tau^{2}-(\lambda-r)^{2}\right)^{-1 / 2} & \text { for } \tau \leq r \\ \{(\tau-\lambda+r)(\lambda+r-\tau)\}^{-1 / 2} & \text { for } \tau \geq r\end{cases}
$$

Extending the domain $\bar{D}_{2}$ of $(\lambda, \tau)$-integration to $\left\{(\lambda, \tau) ; \lambda_{1}^{(2)} \leq \lambda \leq 2 r, 1+|\lambda-r| \leq\right.$ 
$\tau \leq r\}$ and $\left\{(\lambda, \tau) ; r \leq \lambda \leq \lambda_{2}^{(1)}, \lambda-r \leq \tau \leq r\right\}$, it holds from (10.39) and (10.42) that

$$
\begin{aligned}
& (1+r) I_{0}^{6} \\
\leq & C \int_{\left(c_{2} t-r\right)_{+} / c_{2}}^{\left(c_{1} t-r\right) / c_{1}} d s \int_{\lambda_{1}^{(2)}}^{2 r} z_{\mu, \mu}^{(j)}(s, \lambda)^{-1}(1+|\lambda-r|)^{-1} d \lambda \\
& +C \pi \int_{\left(c_{2} t-r\right)_{+} / c_{2}}^{\left(c_{1} t-r\right) / c_{1}}(1+t-s)^{-1} d s \int_{r}^{\lambda_{2}^{(1)}} z_{\mu, \mu}^{(j)}(s, \lambda)^{-1} d \lambda .
\end{aligned}
$$

The first integral is estimated in a similar way as $I_{0}^{6}$ in Case 2

$$
C\left(\Phi_{\mu}(t)\right)^{2} \begin{cases}(1+t+r)^{-1} & \text { for } c_{2} t \leq r \leq c_{1} t \\ \left(1+c_{2} t-r\right)^{-1} & \text { for } r \leq c_{2} t\end{cases}
$$

The second integral is estimated in a similar way as $I_{0,5}^{3}$.

Case 4. $r \leq c_{2} t, r \leq c_{2}(t-s)$.

In this case,

$$
I_{0}^{2} \leq C(1+r)^{-1} \int_{0}^{\left(c_{2} t-r\right) / c_{2}}(t-s) d s \int_{c_{2}(t-s)}^{c_{1}(t-s)} \tau^{-2} z_{\mu, \mu}^{(j)}(s, \tau-r) d \tau .
$$

In the domain of integration,

$$
\tau-r+c_{1} s \geq c_{2} t-r
$$

Then, the integration by parts yields

$$
\begin{aligned}
I_{0}^{2} & \leq C(1+r)^{-1}\left(1+c_{2} t-r\right)^{-\mu} \int_{c_{2}(t-s)}^{c_{1}(t-s)} \tau^{-2}\left(1+\left|c_{j} s-\lambda\right|\right)^{-\mu} d \tau \\
& \leq C(1+r)^{-1}\left(1+c_{2} t-r\right)^{-\mu} \Phi_{\mu}(t) .
\end{aligned}
$$

Extending the domain $D_{2}$ of $(\lambda, \tau)$-integration to $\left[\lambda_{1}^{(2)} \leq \lambda \leq \lambda_{2}^{(1)}\right] \times\left[c_{2}(t-s), c_{1}(t-s)\right]$,

$$
\begin{aligned}
I_{0}^{3} & \leq C(1+r)^{-1} \int_{0}^{\left(c_{2} t-r\right) / c_{2}} d s \int_{\lambda_{1}^{(2)}}^{\lambda_{2}^{(1)}} \lambda^{-1} z_{\mu, \mu}^{(j)}(s, \lambda) d \lambda \\
& \leq C(1+r)^{-1} \sum_{i=1}^{2} I_{0, i}^{3}
\end{aligned}
$$


where $I_{0, i}^{3}$ is defined in Case 3 . Hence,

$$
I_{0}^{3} \leq C(1+r)^{-1} \Phi_{\mu}(t) \sum_{i=1}^{2}\left(1+c_{i} t-r\right)^{-\mu} .
$$

By a similar manner,

$$
\begin{aligned}
I_{0}^{4} & \leq C(1+r)^{-1} \int_{0}^{\left(c_{2} t-r\right) / c_{2}}(1+t-s) d s \int_{\lambda_{1}^{(2)}}^{\lambda_{2}^{(1)}} z_{\mu, \mu}^{(j)}(s, \lambda)^{-1} d \lambda \\
& \leq C(1+r)^{-1} \sum_{i=1}^{2} I_{0, i}^{4},
\end{aligned}
$$

where $I_{0, i}^{4}(i=1,2)$ is defined in Case 3 . Hence,

$$
I_{0}^{4} \leq C(1+r)^{-1} \Phi_{\mu}(t) \sum_{i=1}^{2}\left(1+c_{i} t-r\right)^{-\mu}
$$

Extending the domain of $D_{2}$ of $(\lambda, \tau)$-integration to $\left\{(\lambda, \tau) ; \lambda_{1}^{(2)} \leq \lambda \leq \lambda_{2}^{(1)}, \lambda-r \leq \tau \leq\right.$ $\lambda+r\}$, we also find from (10.35) and (10.42) with $\tau \geq r$ that $I_{0}^{5}$ and $I_{0}^{6}$ are estimated by

$$
\begin{aligned}
& C(1+r)^{-1} \int_{0}^{\left(c_{2} t-r\right) / c_{2}}(1+t-s) d s \int_{\lambda_{1}^{(2)}}^{\lambda_{2}^{(1)}} z_{\mu, \mu}^{(j)}(s, \lambda)^{-1} d \lambda \\
\leq & C(1+r)^{-1} \sum_{i=1}^{2} I_{0, i}^{4} .
\end{aligned}
$$

Therefore, we have proved the assertion (10.33).

\section{References}

1. R. Agemi and K. Yokoyama, The null condition and global existence of solutions to systems of wave equations with different speeds, Series on Advances in Mathematics for Applied Sciences, No.48, World Scientific (1998) 43-86.

2. P. G. Ciarlet, Mathematical elasticity. vol.1; Three dimensional elasticity, Studies in Mathematics and its Applications, 20, North-Holland, Amsterdam, 1988. 
3. M. E. Gurtin, Topics in finite elasticity, CBMS-NSF Regional Conference Series in Applied Mathematics, No.35, Philadelphia: SIAM (1981) 249-255.

4. A. Hoshiga and H. Kubo, Global small amplitude solutions of nonlinear hyperbolic systems with a critical exponent under the null condition, to appear in SIAM Jour. of Math. Analysis.

5. F. John, Plane waves and spherical means applied to partial differential equations, Interscience, New York, 1955.

6. F. John, Finite amplitude waves in a homogeneous isotropic elastic solid, Comm. Pure Appl. Math. 30 (1977) 421-446.

7. F. John, Instability of finite elasticity, Proc. IUTAM Symposium of Finite Elasiticity, Leheigh University, Boston: Martunus Nijhoff (1980) 249-255.

8. F. John, Lower bounds for the life span of solutions of nonlinear wave equations in three space dimensions, Comm. Pure Appl. Math. 36 (1983) 1-35.

9. F. John, Formation of singularities in elastic waves, Lecture Notes in Physics, 195 (1984) 194-210.

10. F. John, Almost global existence of elastic waves of finite amplitude araising from small initial disturbances, Comm. Pure Appl. Math. 41(1988) 615-666.

11. F. John, Nonlinear wave equations, Formation of sigularities, Pitcher lectures in the Math. Sci. Leheigh Univ. American Math. Soc. (1990)

12. S. Klainerman, Weighted $L^{\infty}$ and $L^{1}$ estimates for solutions to the classical wave equation in three space dimentions, Comm. Pure Appl. Math. 37 (1984) 269-288.

13. S. Klainerman, The null condition and global existence to nonlinear wave equations, Lectures in Appl. Math., American Math. Soc. 23(1986) 293-326.

14. S. Klainerman, T. C. Sideris, On almost global existence for nonrelativistic wave equations in 3D, Comm. Pure Appl. Math. 49 (1996) 307-321.

15. T. C. Sideris, The null condition and global existence of nonlinear elastic waves, Invent. Math. 123(1996) 323-342.

16. K. Yokoyama, Global existence of classical solutions to systems of wave equations with critical nonlinearity in three space dimensions, to appear J. Math. Soc. Japan. 